\title{
Nanotechnology-Employed Bacteria-Based Delivery Strategy for Enhanced Anticancer Therapy
}

\author{
Zixuan $\mathrm{Ye}^{1, *}$ \\ Lizhen Liang ${ }^{\prime} * *$ \\ Huazhen Lu' \\ Yan Shen ${ }^{2}$ \\ Wenwu Zhou ${ }^{3}$ \\ Yanan Li (D)
}

'School of Food Science and Pharmaceutical Engineering, Nanjing Normal University, Nanjing, People's Republic of China; ${ }^{2}$ State Key Laboratory of Natural Medicines, Center for Research Development and Evaluation of Pharmaceutical Excipients and Generic Drugs, Department of Pharmaceutics, School of Pharmacy, China Pharmaceutical University, Nanjing, People's Republic of China; ${ }^{3}$ National Experimental Teaching Demonstration Center of Pharmacy, School of Pharmacy, China Pharmaceutical University, Nanjing, People's Republic of China

*These authors contributed equally to this work
Correspondence: Yanan Li

School of Food Science and

Pharmaceutical Engineering, Nanjing

Normal University, Nanjing, People's

Republic of China

Tel +86I505I850598

Email liyanan@njnu.edu.cn

Wenwu Zhou

National Experimental Teaching

Demonstration Center of Pharmacy,

School of Pharmacy, China

Pharmaceutical University, Nanjing,

People's Republic of China

Email dancingzww@I63.com
Abstract: Bacteria and their derivatives (membrane vesicles, MVs) exhibit great advantages for targeting hypoxic tumor cores, strong penetration ability and activating immune responses, holding great potential as auspicious candidates for therapeutic and drugdelivery applications. However, the safety issues and low therapeutic efficiency by single administration still need to be solved. To further optimize their performance and to utilize their natural abilities, scientists have strived to modify bacteria with new moieties on their surface while preserving their advantages. The aim of this review is to give a comprehensive overview of a non-genetic engineering modification strategy that can be used to optimize the bacteria with nanomaterials and the design strategy that can be used to optimize MVs for better targeted therapy. Here, the advantages and disadvantages of these processes and their applicability for the development of bacteria-related delivery system as antitumor therapeutic agents are discussed. The prospect and the challenges of the above targeted delivery system are also proposed.

Keywords: bacteria, nanomaterial, targeted delivery, immune response, membrane vesicles

\section{Introduction}

Due to the abnormal hyperplasia of the vascular system in tumor tissue, a specific anaerobic, acidic microenvironment is formed in tumors. Over the past decades, targeting delivery of therapeutic drugs into cancer with reduced side effects still remains a challenge in cancer therapy. Researchers have made a series of attempts in the drug delivery systems; various viral vectors (e.g., adenovirus, adenoassociated virus), abiotic carriers (e.g., micelles, liposomes, carbon materials, inorganic structures, self-assembled peptide and protein nanostructures) and cell carriers (e.g., bacteria, red blood cells) were developed to achieve active or passive targeting to tumor tissue. ${ }^{1-4}$ Among the cell carriers, bacteria have been explored in cancer therapy for more than a century, including Salmonella, Listeria monocytogenes and Bifidobacterium. Compared with other synthetic carriers, bacteria exhibit multiple advantages, such as (1) the unique ability to preferentially penetrate and colonize anaerobic tumors by an aerotaxis or chemotaxis pathway; ${ }^{5}$ (2) their intrinsic genetic system, which is easily engineered to deliver antitumor agents such as genes or proteins; ${ }^{6}$ and (3) their own immune-stimulating activity, ${ }^{7}$ which allows them to act as adjuvants in tumor immunotherapy. Despite the above advantages, several challenges in bacteria-mediated delivery still exist, such as (1) the risks and safety of bacteria and their derivatives $;^{8-10}$ (2) the active targeting efficiency remains to be enhanced; and (3) expanding the types of drug carried by 
bacteria. ${ }^{11}$ To address these concerns, a nanotechnologyemployed bacteria-based delivery strategy is considered to be an effective strategy to improve bacteria-mediated tumor therapy. In this review, the current developments and their deficiencies of bacteria as therapeutic agents or delivery system are first introduced. Then, we focus on the design strategy of a nanotechnology-employed bacteriabased drug delivery system (e.g., bacteria-derived nanohybrid, bacteria-derived outer membrane vesicles based nano-platforms) and highlight the interaction between bacteria and nanomaterials and the modification strategies to complement or enhance their therapeutic applicability in cancer field. This review can provide more perspectives for the practical medicinal application for a nanotechnology-employed bacteria-based drug delivery system in the future.

\section{Bacteria Used as Carriers in Anticancer Therapeutics}

In the design of a bacteria-based drug-delivery system, the most important thing is the selection of a bacterial strain with specific characteristics to target the tumor area. Until now, various bacterial strains have been used to combat tumors, including Escherichia coli, Clostridium, Listeria monocytogenes, Serratia marcescens, magnetotactic bacteria (MTB) and Salmonella typhimurium. ${ }^{12}$ It is noted that the above bacteria are mainly divided into two types according to application. One is that pathogenic or attenuated bacteria themselves act as therapeutic agents alone, relying on the strong immune stimulation. The other is attenuated or non-pathogenic bacteria mainly acting as a carrier to assist other antitumor therapy.

In 1891, W Busch surprisingly found significant tumor reduction after infection of Streptococcus pyogenes in sarcoma patients. Inspired by this phenomenon, physician W. B. Coley firstly purified and prepared mixed bacteria vaccine (inactivated Serratia marcescens and Streptococcus pyogenes), which has treated thousands of patients with various tumors and mostly exhibited effective tumor suppression. Therefore, attributed to physiological colonization differences between normal and neoplastic tissues, the pathogenic anaerobes were initially developed as immune-stimulating vaccines. The unusually successful case is Mycobacterium bovis BCG, which had been clinically used in postsurgical bladder cancer to prevent cancer recurrence via intravesical infusions of $M$. bovis BCG suspension. ${ }^{13-15}$ The clinical data indicate that bladder cancer recurrence rate decreased significantly after administration of BCG vaccines. ${ }^{15,16}$ However, the immune response of most bacteria-based vaccines is not long-lasting, and is usually accompanied with poor selectivity and serious side-effects. Gradually, the genetically modified and attenuated pathogenic bacteria were developed for safer and longer application (Table 1). Firstly, the attenuated bacteria-based vaccines were carried out by deleting the disease-causing genes to reduce risks, including Aduro's Listeria monocytogenesbased platform, Salmonella typhimurium, ${ }^{17,18}$ etc. Then, the attenuated bacteria were engineered to express molecules, or antigens to specifically enhance the immune antitumor responses. In preclinical study, C. novyi-NT $T^{19}$ induced a strong inflammatory response involving proinflammatory cytokines such as Interleukin-6, granulocyte colony-stimulating factor (G-CSF), macrophage Inflammatory Protein-2, and tissue inhibitor of metalloproteinases 1(TIMP-1) that recruit a substantial amount of immune cells to tumor site to generate a durable adaptive anti-tumor immunity. ${ }^{20,21}$ In clinic, S. typhimurium was modified to express interleukin-2 (IL-2), which showed no toxicity or adverse events. However, there was no evidence of complete response and survival advantage in the single dose clinical Phase I study. ${ }^{22}$ A Listeria-based vaccine vector takes advantage of the intrinsic capacity of the bacterium to forcefully invade antigen-presenting cells (APCs) and engineering expression of shared tumorassociated antigens or unique neoepitopes personalized to each patient. ${ }^{23-26}$ Live attenuated, double-deleted (LADD) Listeria monocytogenes encoded the human mesothelin as a tumor-associated antigen overexpressed in $30-70 \%$ of non-small cell lung cancer (JNJ-757) indicated well tolerated safety in the clinical phase I study. It is noted that the JNJ-757 as monotherapy only induced limited adaptive immune response. Even combined with nivolumab, the clinical best overall disease response with the combination was stable disease in four of 12 patients, which led to not proceeding to Phase $2 .^{27}$ In addition to the above pathogenic bacteria or attenuated pathogenic bacteria, some non-pathogenic bacteria or probiotics including Bifidobacterium, Lactobacillus, and E. coli Nissle 1917 were also applied in antitumor investigation. However, from the preclinical and clinical results, all the pathogenic bacteria or attenuated pathogenic bacteria after genetic modification only induced insufficient tumor suppression, which could not completely kill tumors. Among these non-pathogenic bacteria, the 
Table I Clinical Trials of Bacterial Tumor Therapy (https://www.clinicaltrials.gov/)

\begin{tabular}{|c|c|c|c|c|}
\hline Bacteria & $\begin{array}{c}\text { NCT } \\
\text { Number }\end{array}$ & Phase & Modification & Route/Tumor Types \\
\hline Mycobacterium bovis & & Market & None & $\begin{array}{l}\text { Intravesical infusions/bladder } \\
\text { cancer }\end{array}$ \\
\hline Salmonella typhimurium & NCT0I09963I & Phase I & Knock out msbb, puri, and expressed IL-2 & $\begin{array}{l}\text { Oral administration/Unresectable } \\
\text { hepatic metastasis }\end{array}$ \\
\hline $\begin{array}{l}\text { Salmonella typhimurium } \\
\text { (VNP20009) }\end{array}$ & NCT00004988 & Phase I & Knock out msbb, puri & $\begin{array}{l}\text { Intravenous injection/Advanced } \\
\text { solid tumors }\end{array}$ \\
\hline $\begin{array}{l}\text { Clostridium butyricum CBM } \\
588\end{array}$ & NCT03922035 & $\begin{array}{l}\text { Phase I, } \\
\text { Recruiting }\end{array}$ & None & $\begin{array}{l}\text { Given PO/Hematopoietic and } \\
\text { Lymphoid Cell Neoplasm }\end{array}$ \\
\hline Clostridium novyi-NT & NCT01924689 & Phase I & Eliminate a residential phage carrying $\alpha$-toxin & $\begin{array}{l}\text { Intratumoral Injection/Solid } \\
\text { tumor malignancies }\end{array}$ \\
\hline $\begin{array}{l}\text { Double-Deleted Listeria } \\
\text { Monocytogenes (pLADD) JNJ- } \\
64041809\end{array}$ & NCT02625857 & Phase I & $\begin{array}{l}\text { Delete of two virulence genes from the } \\
\text { L. monocytogenes chromosome-actin } \\
\text { assembly protein }\end{array}$ & $\begin{array}{l}\text { Intravenous administration/ } \\
\text { Metastatic castration-resistant } \\
\text { prostate cancer }\end{array}$ \\
\hline $\begin{array}{l}\text { E. coli Nissle 1917 } \\
\text { Drug: SYNBI89I } \\
\text { Drug: Atezolizumab }\end{array}$ & NCT04I67I37 & $\begin{array}{l}\text { Phase I, } \\
\text { Recruiting }\end{array}$ & $\begin{array}{l}\text { Encode diadenylate cyclase, dihydropyridine } \\
\text { dicarboxylate synthase and thymidylate } \\
\text { synthetase }\end{array}$ & $\begin{array}{l}\text { Intratumoral injection/Metastatic } \\
\text { solid tumors and lymphoma }\end{array}$ \\
\hline
\end{tabular}

engineered E. coli Nissle 1917 (SYNB19891) was first to proceed to Phase 1. Different from other engineered bacteria, SYNB1891 expressed cyclic di-nucleotidesproducing enzymes (diadenylate cyclase) under a tetracycline-inducible promoter (Ptet) which could activate the stimulator of interferon genes (STING) pathway locally in tumor, suggesting a potentially advantageous safety profile and significantly broader set of tools for genetic manipulation. ${ }^{28}$

While the clinical studies of various bacteria or their spores demonstrated that the treatment resulted in reliable colonization in anaerobic tumor tissue, complete suppression of tumor, including its well-oxygenated regions inhospitable to anaerobic bacteria, was still a challenge. Recently, the synergistic combination therapy with bacteria were carried out in clinic to kill tumor cells in both well-oxygenated and hypoxic regions, including chemotherapeutic drugs, immune drugs (NCT03371381). Several clinical data of these combination therapies indicated increased oncolysis and enhanced anti-tumor response compared with the single bacteria therapy (NCT04167137). Thus, the next phase of the development of bacterial therapy involved methods to simultaneously integrate various therapies onto the bacterial therapy, in which the bacteria act as delivery vectors.

\section{Bacteria-Nanoparticle Biohybrids}

The biohybrid concept offers a means to broaden antitumor application of bacteria by integrating live bacteria with abiotic systems such as micro/nanoparticles, allowing them to work simultaneously to achieve advanced levels of functionality beyond that achievable by each component alone. As is known, when given via a systemic injection such as i.v., some living organisms may face some challenges in that it rapidly caused serious systemic inflammatory responses and experienced antibody-mediated clearance. Hence, the hybrid by modifying nanomaterial on the surface of bacteria provides a "shielding" strategy to overcome the systemic inflammation. Moreover, a biohybrid exploits the navigation of targeting bacteria to deliver biomedical cargo to tumor tissue, by assembling drug-loading cargos around the motile bacteria. On the one hand, the biohybrid could serve as a highly flexible chassis to provide broader potential application in a variety of therapeutic combinations, by precisely adjusting the types of loading agents in the coating materials, such as chemotherapeutic drugs, photothermal drugs, photodynamic drugs or immunotherapeutic drugs. Upon the arrival at tumor sites, the smart release of cargos locally inside the tumor would be achieved to 
guarantee maximum efficiency and reduced side effects. Thus, the triggered drug release by the specific tumor environment (including slightly acidic $\mathrm{pH}$, specific enzyme, ROS) could further facilitate the versatile elaboration of these biohybrids. ${ }^{29}$ Herein, we focus on the binding strategies between bacteria and drugloading coating material, their efficiency and scope of application in the anticancer field (Figure 1).

\section{Covalent Bond}

Bacteria as a type of living organism, are convenient to be chemically modified because of various chemical groups on their surface, such as amino groups $\left(-\mathrm{NH}_{2}\right)$ intrinsic to bacteria cell membrane proteins. Some nanoparticles could achieve covalently chemical conjugation by functionalizing with the reactive groups $(-\mathrm{COOH},-\mathrm{CHO})$, which generated amide bond or imine bond to form bacteria-

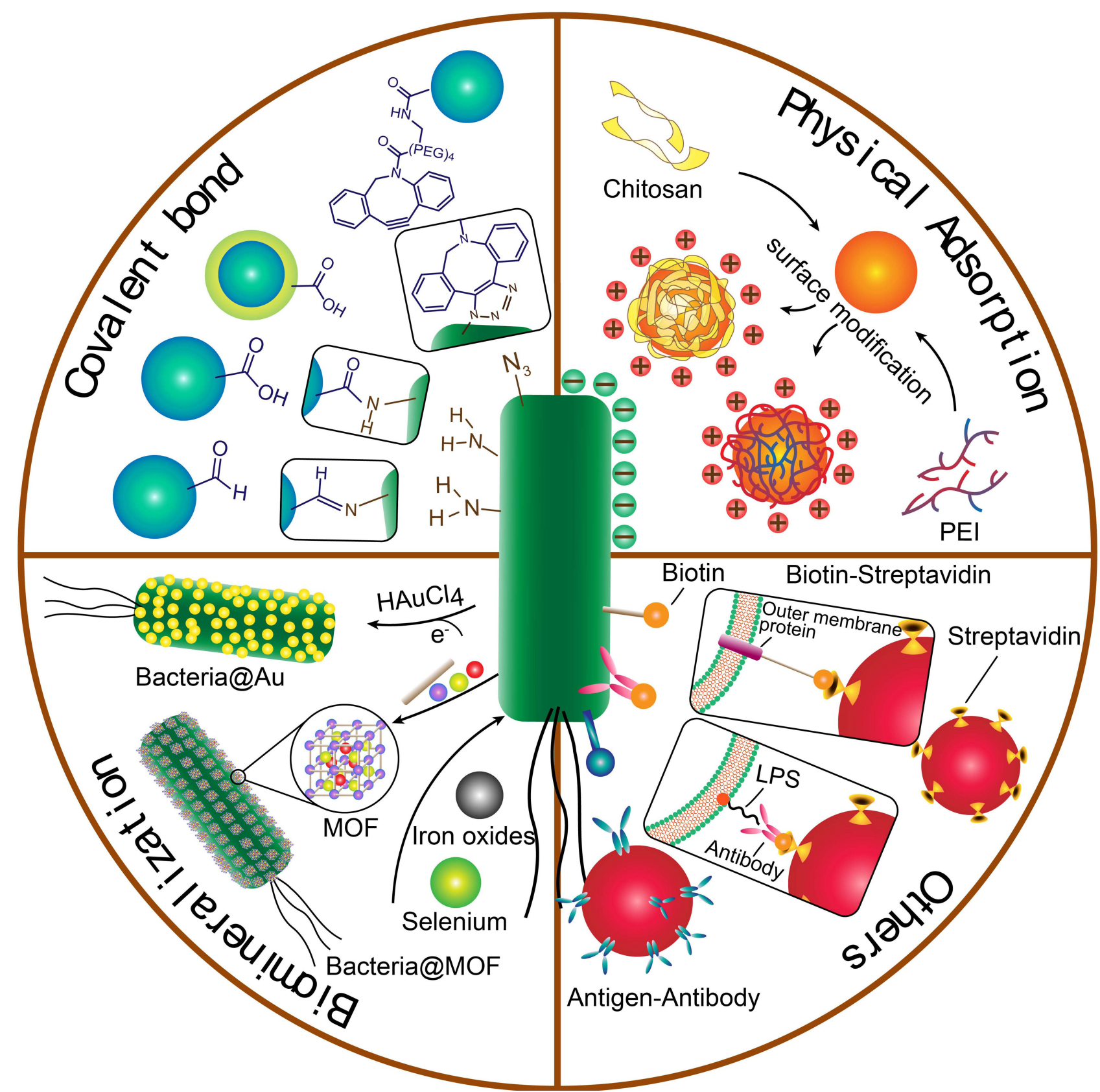

Figure I The illustration of fabrication of bacteria-nanoparticle bio-hybrids via chemical bonds, physical adsorption, biomineralization and other binding forms. Covalent bonds could be formed via reaction of groups on the surface of bacteria and nanoparticles; physical adsorption occurs between negative charged bacteria and positive charged nanoparticles, which are always coated with cationic polymers such as chitosan and PEl; biomineralization is the process of grabbing and turning metal ions into the element metal on the surface of bacteria; other binding forms include the bioaffinity or specific attachment such as biotin-streptavidin affinity and antigen-antibody binding. Abbreviations: PEI, polyethylenimine; LPS, lipopolysaccharides; MOF, metal organic framework. 
nanomaterial biohybrid. $^{30}$ For instance, indocyanine green-loaded nanoparticles were covalently attached to surface of $S$. typhimurium YB1 strain via amide bonds to form YB1-INPs. YB1-INPs maintained superior targeting ability for tumor and photothermal effect, which contributed to 14-times bioaccumulation in tumor for significant tumor elimination without recurrence. ${ }^{31}$ Amide bonds were the most frequent strategy to connect with bacteria by adding carboxyl in the nanoparticles, with superior stability in vivo. Luo et al. ${ }^{32}$ also attached perfluorohexane-loaded poly (lactic-co-glycolic acid) (PLGA) nanoparticles to the surface of anaerobic bifidobacterium longum. This biohybrid could carry the perfluorohexane to deep tumor tissues, enhancing the therapeutic effect of highintensity focused ultrasound therapy. Compared with the amide-bond connection, the imine-bond connection for biohybrid would cause unstable separation in acid tumor microenvironment (TME), due to the hydrolysis of imine bonds in acid solution. However, this strategy could achieve specific separation of nanoparticles from bacteria in TME, which cause no effect on the uptake of nanoparticles by cells. Chen et al. ${ }^{33}$ prepared a photosensitizer-loaded nanoparticles (Zeolite imidazole framework (ZIF-90)), and an imine bond was formed between the aldehyde group of ZIF-90 and the amino group of the bacterium to modify the Shewanella $m R-1$. After moving into acid tumor tissues, the photosensitizerloaded ZIF-90 fall off from the surface of bacteria and achieve photothermal and photodynamic antitumor effect under laser irradiation.

Magnetotactic bacteria can synthesize magnetosomes (magnetic nanoparticles, usually composed of iron oxide) inside their cells, which are aligned longitudinally like compasses to guide magnetotactic bacteria as an excellent targeting carrier. ${ }^{34}$ Magnetococcus marinus strain MC-1, the magneto-aerotactic bacteria, was introduced to transport SN-38-loaded liposomes into hypoxic regions of tumor. The attachment of liposome relied on the covalent bond between the carboxyl of DSPE-PEG-COOH and the amino groups on the bacteria surface. By magnetotactic directional control towards the hypoxic regions of tumor, the mean tumor targeting ratio $(>50 \%)$ was achieved to enhance the efficiency of the targeted chemotherapy. Many other therapeutic modalities could also benefit from this delivery strategy, including delivering the radio-sensitizers or photodynamic sensitizer into the tumor hypoxic regions, which enhanced the radiotherapeutic or photothermal treatments. Another study ${ }^{35}$ further evaluated the effect on the swimming speed of magnetotactic bacteria after attaching the liposomes to the surface. Their research evidenced a $27 \%$ decline of the swimming speed and higher velocity at the same magnetic field for the biohybrid, which proved that magnetotactic bacteria could still assist the nanoparticle to overcome diffusion resistances in solid tumors by chemical connection.

In addition to the direct attachment, there also exists another chemical modification strategy in bacteriananomaterial hybrids. Kuru et al. ${ }^{36}$ exploited a one-sizefits-all strategy to modify the surface of bacteria. They found unnatural D-amino acids of various sizes and functionalities could be incorporated into peptidoglycan (PG) on the bacteria. By introducing the 7-hydroxycoumarin 3-carboxylic acid (HCC-OH) and 4-chloro-7-nitrobenzofurazan (NBD-Cl), to a D-amino acid backbone, these non-toxic D-amino acids preferably label the sites of PG synthesis, enabling the spatiotemporal tracking of cell wall. $^{36}$ This strategy provided more chemical sites for the nanomaterials to attach on. A typical way is to first modify the bacteria with azide groups and modify nanomaterials with alkyne-strained groups. Then both functional groups could form stable triazole bonds by click reaction. Moreno et al. attached drug-loaded mesoporous silica nanoparticles to the surface of Escherichia coli bacteria, which achieved higher penetration in tumoral matrices and homogeneous distribution of therapeutic agents inside tumors. ${ }^{37}$

Compared with other bioconjugation techniques, the covalent bonds are stronger with the bond dissociation enthalpy above $300 \mathrm{~kJ} \cdot \mathrm{mol}^{-1}$. Hence, during the in vivo navigation, the biohybrid would likely remain stable until arriving at tumors. Future studies need to further investigate whether nanomaterials undergo detachment from bacteria in the cellular environment or bacteria /nanomaterial complexes themselves can be internalized by nonphagocytic cells.

\section{Physical Adsorption}

Apart from the chemically covalent modification, the physical adsorption was also introduced to fabricate bacteriananoparticle biohybrids, including van der Waals forces, electrostatic forces, hydrogen bonds, and hydrophobic effects, etc. ${ }^{38}$ As is known, the surface of bacteria displays negative potential, so there appeared some strategies to reverse the nanomaterial potential from negative to positive via adding cationic polymers or being protonated. Then the hybrid could be formed by electrostatic 
adsorption forces. ${ }^{38}$ Polyethylenimine (PEI), as a cationic polymer, can be combined with nanoparticles by surface modification, then absorbing to the surface of bacteria. $\mathrm{Wu}$ et al. ${ }^{38}$ coated a photosensitizer-loaded lipid nanoparticle onto the surface of E. coli, with the above strategy (PEI, $600 \mathrm{Da}$ ). This multifunctional hybrid achieved better invasion ability for cancer cells and efficient light-mediated cancer killing (Figure 2). Similarly, Hu and Chen et al. ${ }^{39,40}$ constructed plasmids encoding the vascular endothelial growth factor receptor (VEGFR2) gene and antigenic gene as DNA vaccine. By electrostatic self-assembly of $\beta$ cyclodextrin-PEI and pDNA as nanoparticles, the DNA nano-vaccine was decorated on the surface of invasive Salmonella by electrostatic interaction. The cytotoxic $\mathrm{T}$ lymphocytes (CTLs) are activated for immunotherapy, which further inhibits the formation of tumor blood vessels by affecting the VEGFR2 pathway, leading to a thorough tumor suppression. In fact, bacteria themselves have certain immune-stimulating activity, acting as adjuvants, so that a DNA vaccine/bacteria hybrid could amplify the immune activation effect in TME. Other cationic polymers such as chitosan or cationic peptides may also be suitable for the binding of complexes. Except for cationic polymer, protonation can also make the nanoparticles positively charged. Luo et al. ${ }^{41}$ used protonated oleic acid to wrap the imaging agent-loaded nanorods. With the positively charged surface, the nanorods were combined with anaerobic bifidobacterium breve UCC2003 through electrostatic interaction. Through the targeting of bacteria, the imaging agent concentrated at tumor sites to increase the

A

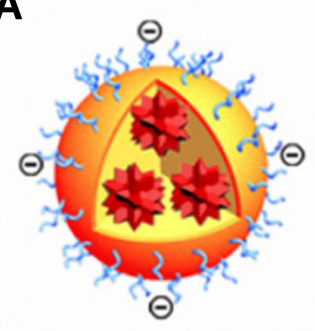

TDNP

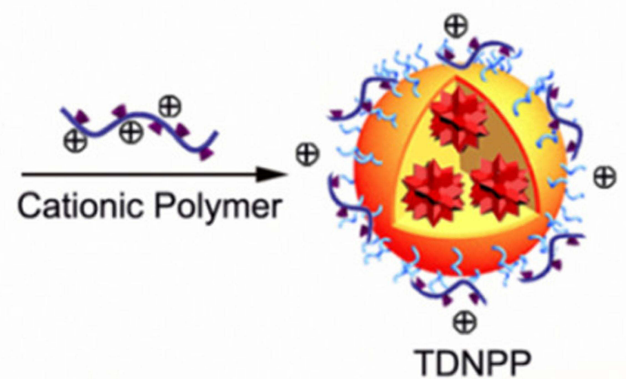

TDNPP

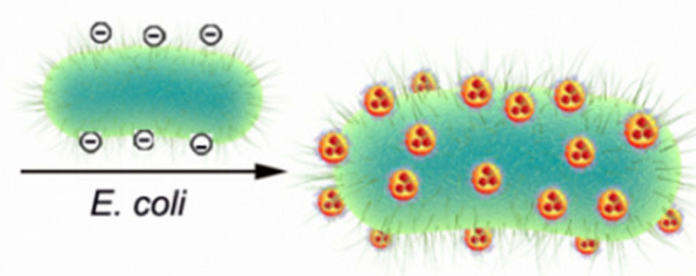

TDNPP-E. coli

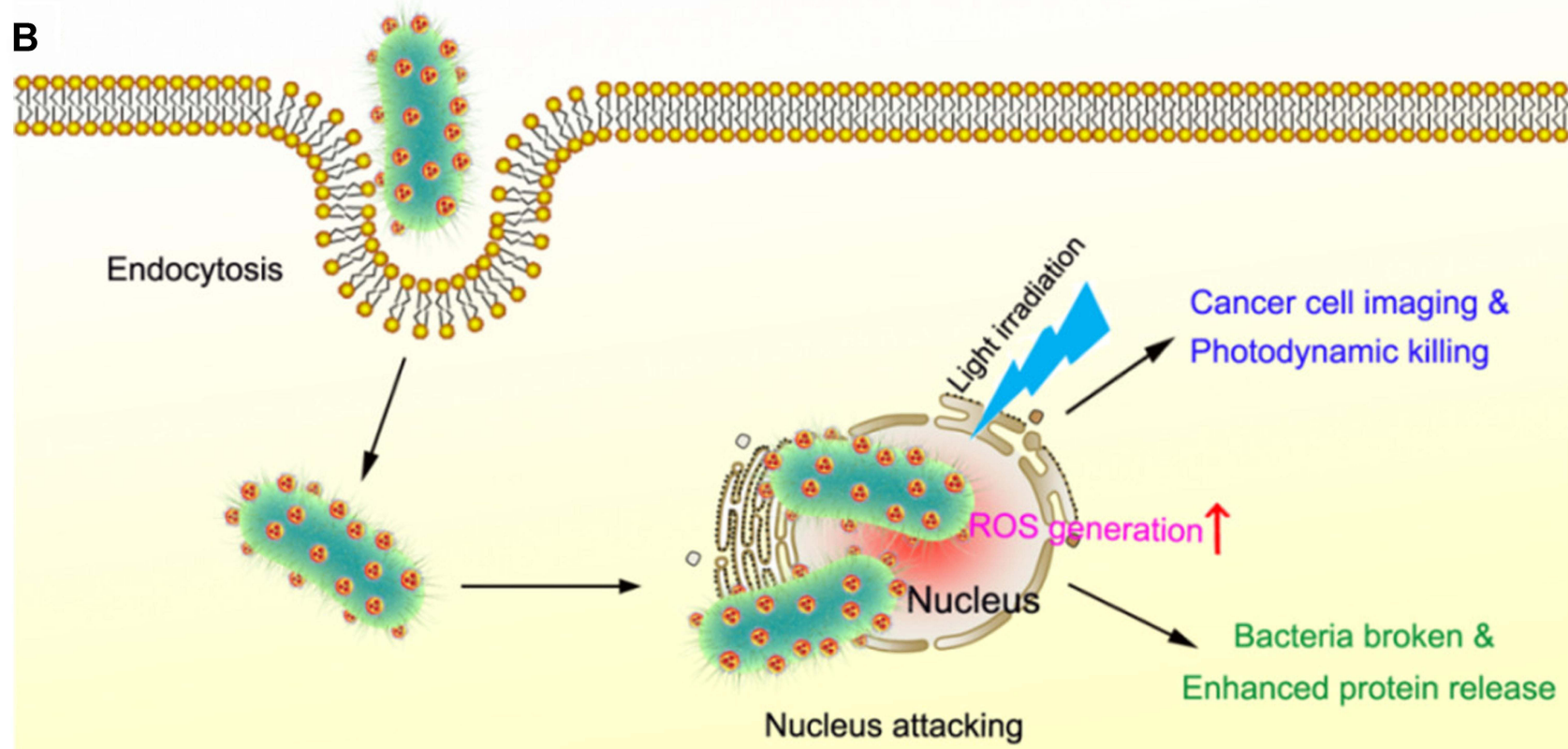

Figure 2 (A) Process of PEl employed photosensitizer nanoparticles (TDNPP)-coated live $E$. coli. (B) Intracellular trafficking of nanoparticle-coated live E. coli and photosensitizer delivery. Adapted with permission from Wu M, Wu W, Duan Y, Li X, Qi G, Liu B. Photosensitizer Bacteria Biohybrids Promote Photodynamic Cancer Cell Ablation and intracellular Protein Delivery. Chem Mater. 2019;3I(I8):7212-7220. Copyright 2019 American Chemical Society. ${ }^{38}$ 
fluorescence intensity in tumor. This strategy could be applied in various bacteria/nanomaterial construction.

In addition, supramolecular self-assembly is a physical process in which molecules spontaneously connect into molecular aggregates with stable structure on the membrane surfaces, relying on the intermolecular forces of non-covalent bonds. Recently, a smart coating method with lipid membranes based on interfacial supramolecular self-assembly was found to keep bacteria unbroken during the first $4 \mathrm{~h}$ in the stomach. Then, nearly $90 \%$ of the bacteria disassembled from the coating after 4 $\mathrm{h}$ following arrival in the intestines, which in turn results in the recovery of their inherent properties. This intelligent delivery system using FDA approved materials significantly increased the preservation and bioavailability, providing a smart delivery strategy for the precise targeting to intestines. ${ }^{42}$ Hence, if antitumor drug was loaded inside the bacteria, this supramolecular self-assembly strategy could be utilized to target and treat intestinal tumors.

In general, the passive adsorption through non-covalent bonds, such as van der Waals forces or electrostatic forces is easy to form. However, the conjugations may suffer from poor stability in vivo, especially in plasma. In addition, it should be taken into account that if bacteria proliferate in vivo, the absorbed nanoparticles may fall off the surface. Once the outer layer is lost, most bacteria exposed in the blood may trigger a type of inflammatory storm. Therefore, the non-pathogenic probiotics as therapeutic agent vectors may be a better choice in this kind of physically adsorbed hybrid.

\section{Biomineralization Process}

Biomineralization is a process in which the living organisms translate mineral materials into their biological matrix. It is interesting that some bacteria could biomineralize nanoparticles by grabbing and turning metal ions into the element metal through a biological enzymatic process. Scientists have constructed many kinds of bacteriainorganic composites via this biomimetic mineralization strategy, which have been applied in many fields such as sensing, imaging and catalysis. However, there still exists great potential in therapeutic delivery. Bacteria as a tumortargeting live organism, could also be endowed with various functions in antitumor therapy in this way. First, biomineralization nanoparticles on the surface of bacteria would not affect the activity of bacteria as a tumortargeting navigation. Thus, the bacteria-based therapeutic platforms by biomineralization could provide a synergetic option to achieve the tumor-targeting capacity and suppress tumor by drug-loaded nanoparticles. Various materials have been used in biomimetic mineralization on the surface of bacteria, including $\mathrm{ZnS}$, silica, selenium, iron oxides, calcium phosphate, gold nanoparticles and metalorganic frameworks (MOF).

First, many metal and metallic oxides have been proved as photothermal agents for photothermal therapy such as tetrapyrrolic derivatives of Palladium (II)(WST11), Lu(III) (Lutex), and Sn(IV) (Purlytin), thereby applying specific bacteria to biosynthesize the photothermal agents on the surface could increase the targeting ability of PTT. ${ }^{43}$ Chen et al. introduced the facultative anaerobic bacterium Shewanella oneidensis MR-1 to reduce sodium tetra-chloropalladate $\left(\mathrm{Na}_{2} \mathrm{PdCl}_{4}\right)$ into Pd nanoparticles on its surface. ${ }^{33,44}$ This selfmineralized photothermal bacterium was reported to possess preferential tumor-targeting ability and superior photothermal properties. ${ }^{45}$ Wang et al. biosynthesized gold nanoparticles on the surface of Shewanella algae K3259. This hybrid not only promoted the targeting of photodynamic therapy of gold nanoparticles, but also accelerated bacterial metabolism to improve the production of antineoplastic tetrodotoxin for antitumor therapy by transferring photoelectrons produced by AuNPs into bacterial cytoplasm. ${ }^{46}$ To extend the loading types, Yan et al. ${ }^{47}$ constructed a bacteria@MOF hybrid by biomineralized Escherichia coli (MG1655) with a zeolitic imidazolate framework-8 layer (MOF). Inside MOF, a photosensitizer (chlorin e6, C) and a chemotherapeutic drug (doxorubicin, D) were loaded which exhibited synergistically excellent therapeutic efficacy (Figure 3). Based on this approach, more application of various therapies could be broadened via loading different drugs into the drug delivery carriers biosynthesized on the surface of bacteria, such as MOF, silica nanoparticles, calcium carbonate or calcium phosphate nanoparticles.

The biomineralization process conjugating the bacteria with some inorganic nanoparticles gives the drug delivery systems some unique functions such as magnetic, photothermal conversion ability. However, certain amino acids on the surface of the living agents limited the universality of the strategy and the morphology needs to be further studied. $^{48}$

\section{Other Binding Forms}

Other methods of attachment on the bacteria have also been used such as bioaffinity or specific attachment (e.g., antibody-antigen), ${ }^{49,50}$ in the bacteria-nanoparticle 

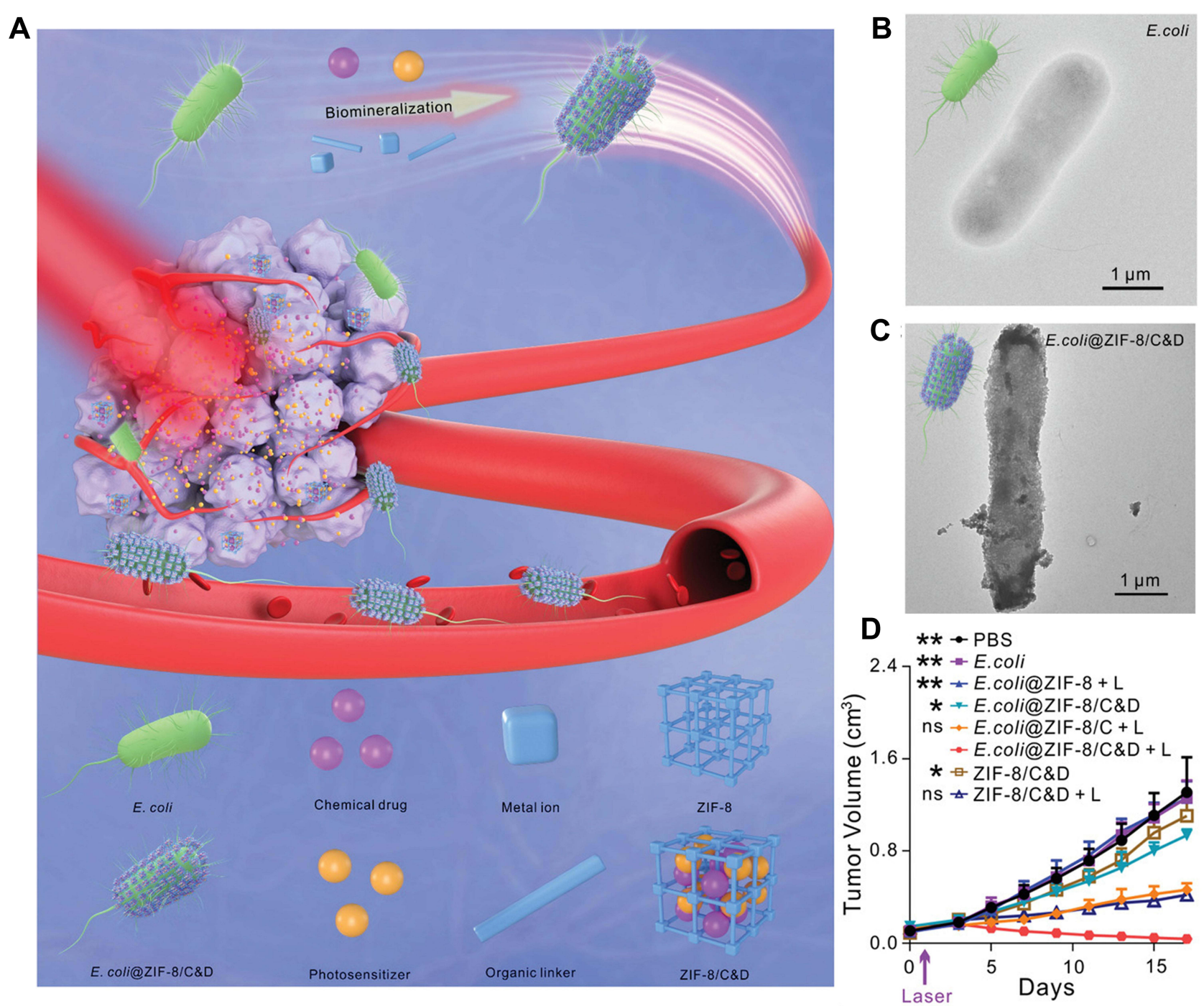

Figure 3 (A) Biomimetic mineralization of tumor-targeting E. coli by zeolitic imidazolate framework-8 (ZIF-8) for the delivery of therapeutics. (B-C) TEM images of primary E. coli and E. coli@ZIF-8/C\&D. (D) Tumor growth curves of 4TI tumor-bearing mice with different treatments ( ${ }^{*} p<0.05$, **p $<0.01$ ). Adapted with permission from Yan S, Zeng X, Wang Y, Liu BF Biomineralization of Bacteria by a Metal-Organic Framework for Therapeutic Delivery Adv Healthcare Mater. 2020;9(I2):e2000046. @ 2020 WILEYVCH Verlag GmbH \& Co. KGaA, Weinheim. ${ }^{47}$

biohybrids. These relatively close binding forces are the specific interaction forces already existed in the organism.

First, the affinity between streptavidin and biotin is a protein-ligand interaction, one of the strongest binding forces in nature. Biotin could be closely captured by a tetrameric biotin-binding protein, streptavidin, which is widely used in the targeting modification on the drug delivery carrier. $^{51,52}$ In this strategy, biotin molecules were always bound to the outer membrane of bacteria by incubating antibody-modified biotin with bacteria for $1 \mathrm{~h}$, and streptavidin was covalently attached on the surface of the nanoparticles. Then the hybrid was formed through the co-incubation of biotin-labeled bacteria and streptavidincoated nanoparticles. Sahari et al. treated the E. coli
$M G 1655 m$ with goat polyclonal anti-lipid A LPS antibody labeled with biotin, and then streptavidin-coated polymeric microparticles were decorated on the surface of bacteria. ${ }^{53}$ Moreover, poly(lactic-co-glycolic acid) nanoparticles were displayed on the surface of S. typhimurium VNP20009 using a similar strategy. They found the nanoparticle conjugation did not impede bacteria's intratumoral transport performance, even enhancing retention and distribution of nanoparticles in solid tumors by up to a remarkable 100fold $^{54}$ (Figure 4). Uthaman et al. have modified the facultative anaerobic Salmonella typhimurium following the genetic transformation to express biotin in order to interact with streptavidin conjugated microbeads. Via a specific interaction between the biotin on the bacteria and 
A

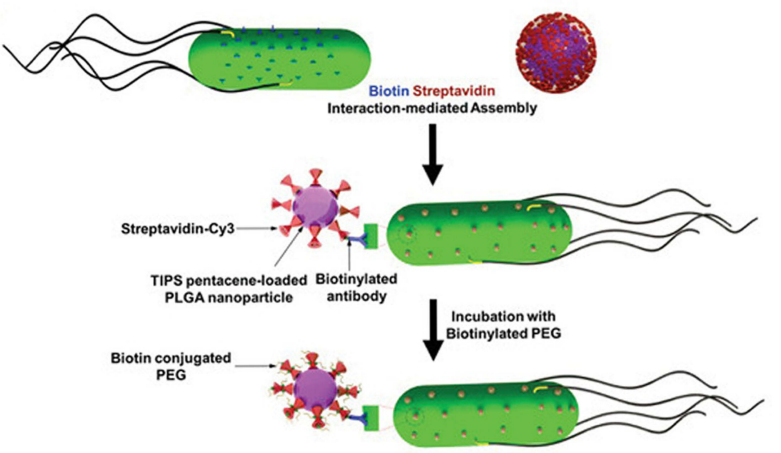

C

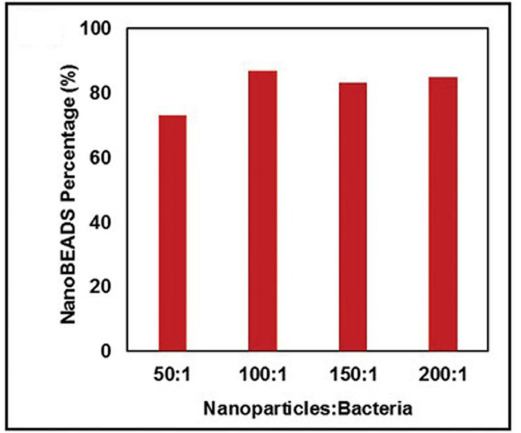

D

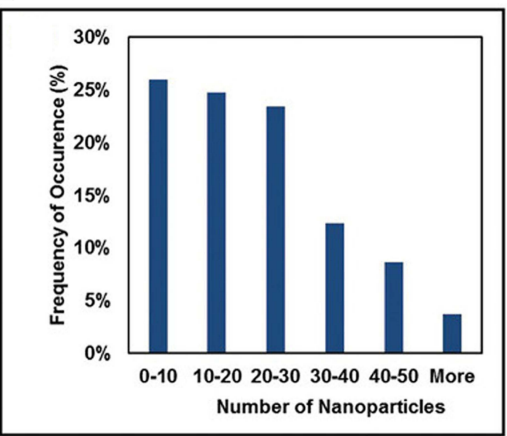

B

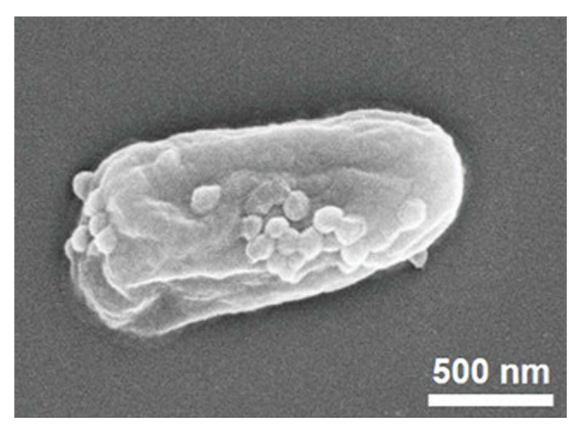

$\mathbf{E}$

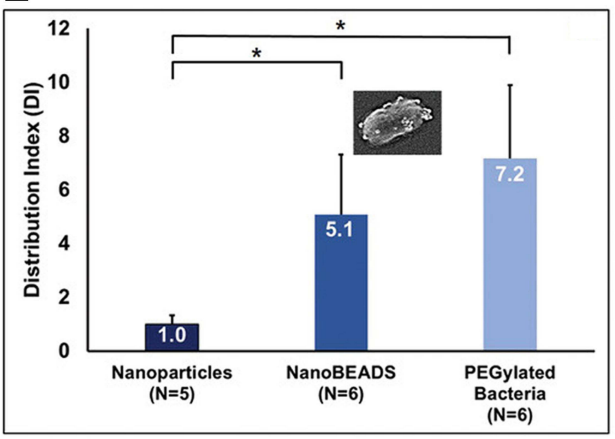

Figure 4 The development of bacteria-enabled autonomous drug delivery system (NanoBEADS) via biotin-streptavidin conjugation. (A) Each NanoBEADS agent is constructed by conjugating several streptavidin-coated PLGA nanoparticles with a tumor targeting biotinylated-antibody coated S. typhimurium VNP20009, using streptavidin-biotin noncovalent affinity-based bonds. NanoBEADS assembly was followed by incubation with mPEG-biotin to quench residual streptavidin binding sites on the nanoparticles. (B) A representative SEM image of a NanoBEADS agent. (C) Percentage occurrence of NanoBEADS formation at various nanoparticle:bacteria ratios used for NanoBEADS construction. (D) Distribution of nanoparticle loading of NanoBEADS agents constructed at nanoparticle to bacteria ratio of I00:I. (E) Distribution index (DI) of PLGA nanoparticles, NanoBEADS, and PEGylated bacteria in 4TI tumors. Each NanoBEADS agent carries an average of 22 nanoparticles, thus, it enhances the intratumoral transport of nanoparticles by up to $\approx 100$-fold $\left({ }^{*} \mathrm{p}<0.05\right)$. Adapted from Suh S, Jo A, Traore MA, et al. Nanoscale Bacteria-Enabled Autonomous Drug Delivery System (NanoBEADS) enhancesintratumoral transport of nanomedicine. Adv Sci. 2019;6(3):I80I309. @ 2018 The Authors. Published by WILEY-VCH Verlag GmbH \& Co. $\mathrm{KGaA}$, Weinheim. This is an open access article distributed under the terms of the Creative Commons CC BY license. ${ }^{54}$

streptavidin on the HA beads, both chemotactic and biological targeting towards breast tumor cells further enhanced the targeted antitumor therapy. ${ }^{55}$ In addition, the number of bacteria attached on the surface of the microbeads could be easily controlled which ensured that the net self-propelling force of all bacteria enables the microbeads to move in a single direction.

Apart from the streptavidin-biotin affinity, the antibody-antigen interaction was also designed for the functional NPs delivery. The nanoparticles could be fabricated by modifying with a layer of monoclonal antibody that is targeted to specific bacteria. According to the principle, Luo et al. ${ }^{41}$ first injected the $C$. difficile spores into tumors, followed by the injection of the antibody-NP to specifically target the germination of the $C$. difficile spores (an antibody-guiding approach). Hence, due to this highly specific antibody-antigen, the bacteria itself can act as guiding markers to attract nanomedicines into tumors and form hybrids in vivo.

\section{Future Opportunities for Bacteria-Nanoparticle Biohybrids Against Cancer}

Various nanomaterials have been investigated to design the bacteria/material hybrid drug delivery system for better antitumor therapy. Among them, the most common ones are liposomes, micelles, etc., which have significant advantages in drug loading and delivery. ${ }^{56,57}$ In addition, polyethylene glycol-modified nanoparticles also act as possible candidates, attributed to flexible modification and the high biocompatibility of polyethylene glycol. ${ }^{58-60}$ Moreover, a variety of other cargo nanomaterials (e.g., polycaprolactone, alginates, chitosan, polystyrene, and cellulose) are also employed. Generally, aside from the attachment in our review, the cargo shapes, attachment density, nonhomogeneous patterned attachment of these nanomaterials on the surface of the bacteria (e.g., Janus-type surfacepatterned coverage) will further affect the bacterial 
swimming speed and sensing ability, thus affecting the tumor targeting ability. According to the reports, all the hybrids after modification could achieve the nanomaterials propulsion speeds ranging from $0.5 \mu \mathrm{m} / \mathrm{s}$ to $30 \mu \mathrm{m} / \mathrm{s}$. How these factors influence the movements of hybrids are in the previous reported review. ${ }^{61}$

In the future, the biohybrid microrobotic system still has many challenges. First, as an effective drug delivery carrier, it must be able to release the drug precisely in a controlled manner at the tumor area in response to the specific conditions in the tumor microenvironment, such as acidic environment, high expression of glucuronidase and matrix metallase, light or ultrasound, etc. Therefore, more intelligent nanoparticles reported could be applied into this hybrid system. Second, it is necessary to avoid the uncontrollable proliferation of bacteria in the body, because the induced autoimmune reaction could result in severe side effects and even death. Although such risk cases lead scientists to turn to attenuated bacteria and nonpathogenic strains, ${ }^{62,63}$ the lipopolysaccharide and other components on the surface of bacteria are still regarded as risky. Recently, the advanced development of synthetic biology provides various strategies to control the retention of bacteria in the body by designing an auxotrophic bacterium, ${ }^{28}$ or constructing a suicide circuit inside the bacterial cells, ${ }^{64,65}$ which step forward in meeting regulatory requirements. It is noted Fan et al. constructed chromosome-free cell called SimCells (simple cells) from Escherichia coli, Pseudomonas putida, and Ralstonia eutropha, via double-stranded breaks made by heterologous I-CeuI endonuclease and the degradation activity of endogenous nucleases. This method could effectively prevent the infinite proliferation of bacterial carrier in the body. ${ }^{66}$ In the future, the engineering of bacteria cells with better safety and even expressing protein drugs could be combined with nanomaterials, trying to gather all the characteristics of an ideal drug delivery system in one bacterium.

\section{Bacteria-Derived Outer Membrane Based Nanoplatforms}

Bacterial outer membrane vesicles (MVs) are secreted by Gram-negative bacteria with particle sizes ranging from 20-400 $\mathrm{nm}$ that participated in diverse biological processes, including horizontal gene transfer, the export of cellular metabolites and cell-to-cell communication. ${ }^{67-69}$ Although other synthetic nano-vectors exhibit similar size, the biological structure and function of MVs endowed it with innate biocompatibility, large drugloading space, high physicochemical stability ${ }^{70}$ and the inherent ability to communicate with cells. In addition, the structure and function of MVs vary from different species. Some MVs have been reported to have natural targeting abilities or specifically internalized by endocytosis. For example, the MVs derived from Escherichia coli were shown to successfully penetrate stratum corneum and accumulate in dermis, having the superior targeting and infiltration in melanoma spheroids; ${ }^{71} \mathrm{MVs}$ obtained from Salmonella and Shigella contain adhesion which could be recognized, endocytosed, and digested in gastrointestinal cells without any targeting modification, providing a targeted delivery therapy for the colon cancer. ${ }^{72}$ On the basis of previous study, bacterial MVs have been successfully used to load different kinds of antitumor drugs with various structures, hydrophobicity, charges and solubility, such as DNA, RNA, paclitaxel, Indocyanine Green (ICG) and TNF-related apoptosis inducing ligand (TRAIL), which had covered chemotherapy, thermo-therapy and immunotherapy. ${ }^{73-83}$

It is noted that bacterial MVs contain multiple bacteriaderived immunostimulatory components, such as lipopolysaccharide (LPS), outer membrane proteins, DNA, RNA and lipoproteins. ${ }^{67,84}$ This realization attracted extensive investigation into whether the treatment of MVs alone could offer comparable pharmacological antitumor benefits. However, the serious systemic inflammatory responses and rapid clearance were found when given to mice via intravenous injection. To expand its application, different strategies have been explored to construct better drug delivery platforms in anticancer immunotherapies. ${ }^{85-87}$ In this part, we mainly focus on the design strategies for bacterial MVs as drug vehicles for better antitumor effect, including genetic manipulation of biosynthesis to produce endogenous species or attaching exogenous species to membrane surface (Figure 5).

\section{Engineering MVs via Genetic Manipulation}

With the advances of synthetic biology, researchers have introduced non-native materials to bacteria to augment therapeutic function. It is highly likely that many of these materials will unwittingly appear within MVs. For example, the incorporated material expressed on the bacterial membrane may be packaged into the MVs for secretion. Taking advantage of this bacteria engineering technique, the MVs functionalization could be achieved. 


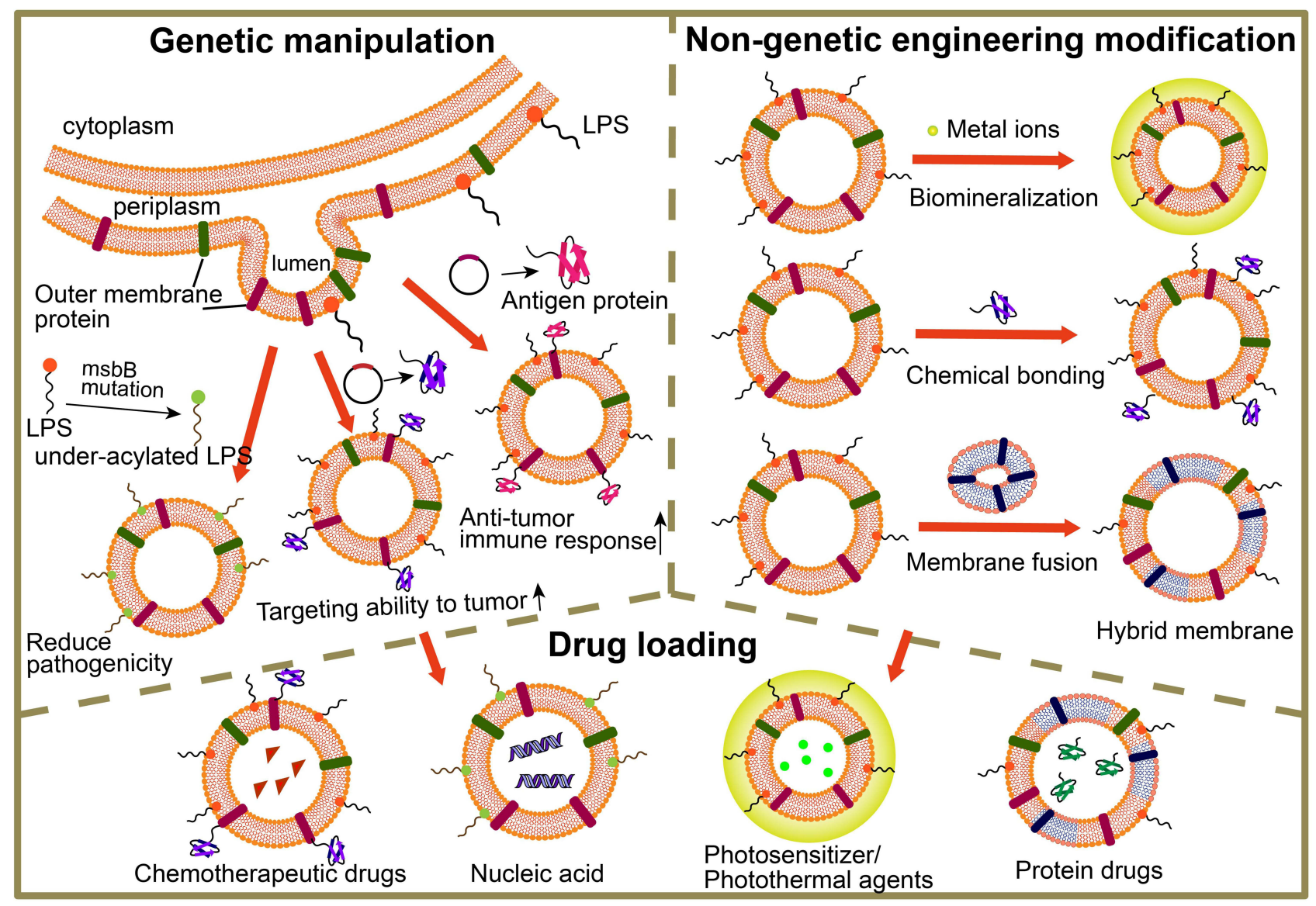

Figure 5 The illustration of MVs engineered by genetic and non-genetic method for drug loading. Genetic manipulation was taken for reducing pathogenicity, enhancing targeting ability to tumor and improving anti-tumor immune response; non-genetic engineering modification including biomineralization, chemical bonding and membrane fusion; the functionalized MVs could load drugs such as chemotherapeutic drugs (e.g., doxorubicin, paclitaxel), nucleic acid (e.g., DNA, siRNA), photosensitizers/ photothermal agents (e.g., ICG) and protein drugs (e.g., TRAIL).

Abbreviation: LPS, Lipopolysaccharides.

Herein, we will discuss how techniques such as genetic engineering can be exploited for the broader application of MVs as targeted vehicles towards tumors.

\section{Reduce Pathogenicity of MVs as Drug Carrier}

The bacteria MVs still had safety concerns that limited their clinical application. In addition to vaccines, to be widely employed in the development of carriers for therapeutic agents, various strategies were explored to reduce their pathogenicity or toxicity. ${ }^{8}$ Firstly, the MVs used as carriers were mostly obtained from non-pathogenic strains. Then, nitrogen cavitation method was exploited to eliminate intracellular components to form double-layer MVs from E. coli BL21, thus further reducing the toxicity. To better act as safe delivery system, the outer membranes of MVs were also manipulated. As we know, the components of lipopolysaccharide (LPS), bacterial endotoxin-lipid A, would cause serious inflammatory responses if injected systemically. Hence, the genetically mutant bacteria strains to modify lipid A were engineered. For example, E. coli K-12 W3110 strain carrying an msbB mutation, was shown to produce under-acylated LPS and thus exhibits reduced endotoxicity toward human cells compared with E. coli strains that produce hexa-acylated LPS. Additionally, the shorter length of o-polysaccharide in LPS further reduces immunogenicity. ${ }^{89}$

\section{Improve Targeting Ability to Tumor}

To effectively treat cancers, the MVs have been developed to target tumor tissue and enhance the therapeutic function via the design of expressing targeting ligands on the surface. As reported, the common proteins with selective localization on surface of MVs includes ClyA, ${ }^{90}$ hemoglobin protease $(\mathrm{Hbp}){ }^{91}$ and outer membrane protein (Omp) A/C/F. Among that, there is a size limitation of surface display protein by fusing with $\mathrm{Hbp}^{90}$ Both of the $\mathrm{N}$ - and 


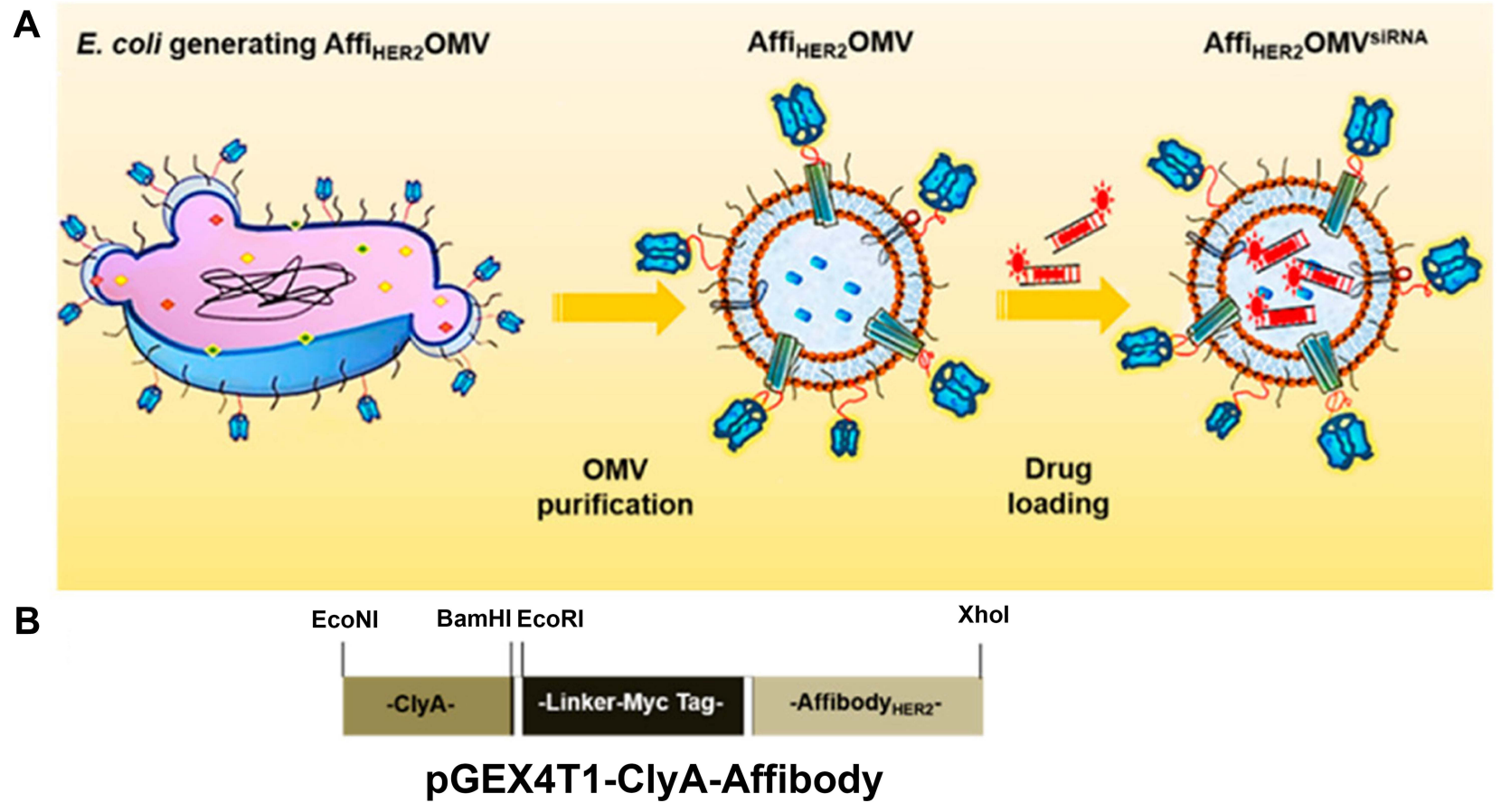

C

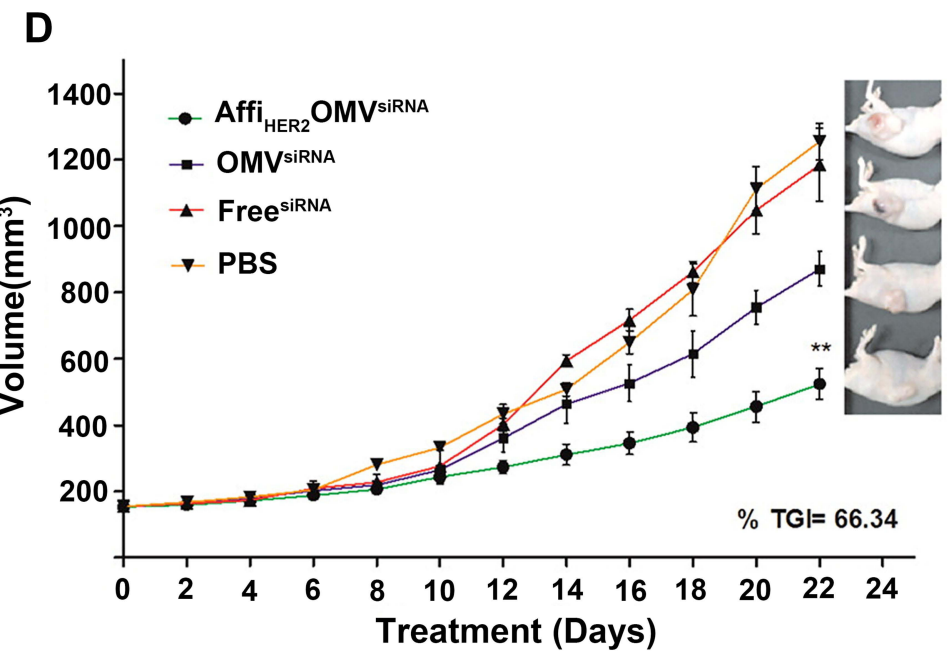

Figure 6 (A) Schematic representation of OMVs expressing HER2-specific affibody (Affi $\mathrm{HER}_{2} \mathrm{OMV}$ ), purified after vesiculation from the parent bacteria and further loaded with siRNA-TAMRA (Affi $\mathrm{HER}_{2} \mathrm{OMV}^{\text {siRNA-TAMRA }}$ ). (B) Schematic representation of the pGEX4TI-ClyA-Affibody construct. (C) Whole-body in vivo imaging revealed accumulation of $\mathrm{Affi}_{\mathrm{HER} 2} \mathrm{OMV}$ siRNA-Cy5.5. The circles (red) indicate the tumor position. (D) Tumor growth inhibition (TGI) was monitored in HCC-I954 xenografts, and $\mathrm{Affi}_{\mathrm{HER} 2} \mathrm{OMV} \mathrm{V}^{\text {siRNA }}$ exerted potent antitumor effects compared with all controls (**p <0.0I). Adapted with permission from Gujrati $\mathrm{V}$, Kim S, Kim SH, et al. Bioengineered Bacterial Outer Membrane Vesicles as Cell-Specific Drug-Delivery Vehicles for Cancer Therapy. ACS Nano. 2014;8(2):1525-I537. Copyright 2014 American Chemical Society. ${ }^{86}$

C-terminals of OmpA/C/F are located on the medial side of the outer membrane, and the insertion of exogenous elements into the middle of the protein may influence the protein structure and folding, especially for the transmembrane proteins. ${ }^{92,93}$ In current studies, ClyA was often selected as the anchor site for surface modification. Recent studies reported that genetic fusion between recombinant polypeptides and the $\mathrm{C}$ terminus of ClyA in bacteria results in a specific display of protein on the surface of MVs, which could modify the physicochemical property of MVs surfaces. ${ }^{94}$ For example, Vipul Gujrati ${ }^{86}$ utilized the addressing ability of ClyA to display a high-affinity anti-human epidermal growth factor receptor 2 (HER2) affibody in the MV surface for the purpose of targeted delivery of therapeutic siRNA targeting kinesin spindle protein (Figure 6). Gao et al. $^{95}$ exploited and expressed RGD4C-EGFP at the 
C-terminus of ClyA on the surface of MVs, to monitor and enhance the tumor targeting by specifically identifying and interacting with Integrin $\alpha_{v} \beta_{3}$ on tumor cell. After packing DOX into the above MVs, they showed promising potential in tumor growth inhibition. Overall, despite some concerns over gene transfer and contamination, genetic manipulation represents a highly accessible strategy for the presentation of flexible targeting function with MVs.

\section{Enhance the Immune Response}

It has been reported that MVs could effectively activate immunotherapy for cancer treatment. Two MVs-based vaccines, Bexsero and MeNZB, have been approved for treatment against meningococcal group $\mathrm{B}$ infections, which highlighted the potential in tumor vaccines. Based on the PAMPs inside MVs, they gradually developed as cancer vaccine adjuvants to stimulate dendritic cell (DC) maturation and promote cytokine release. Besides Toll-like receptors (TLR) agonists (TLR4, TLR2, TLR5 ${ }^{96-99}$ ), the STING agonists were also found in MVs components to produce the strong innate DC stimulation and type I interferon (IFN) secretion, such as the natural stimulator cyclic-dinucleotides (CDNs) from $\mathrm{MVs}^{88}$ Recently, genetic engineering MVs could exhibit exogenous antigen protein, provoke antigen-specific immune response and combat tumor, such as ovalbumin fragment ${ }^{100}$ and basic fibroblast growth factor. ${ }^{101}$ The tumor antigen HPV16E7 was embedded on the surface as well as in the lumen of E. coli-derived MVs through the location ability of ClyA. ${ }^{102}$ This kind of strategy provided a novel vaccine delivery vector, which could be used to deliver more neoantigens to induce specific antitumor immune response effectively. Cheng et al. further employed the protein Plugand-Display system to display various target antigens, including a SpyTag $(\mathrm{SpT}) /$ SpyCatcher $(\mathrm{SpC})$ pair $^{103}$ and a SnoopTag $(\mathrm{SnT}) /$ SnoopCatcher $(\mathrm{SnC})$ pair,${ }^{104}$ in which the protein tag can spontaneously bind to the protein catcher through isopeptide bond formation, by expressing the protein catchers as fusion proteins with ClyA, various tumor antigens linked to protein tags can be rapidly displayed on the MVs surface, eliciting a long-term immune memory in vivo. ${ }^{105}$

Besides the potential in tumor vaccine, the modified MVs were also applied in other immunotherapies. Tumor environment is a complex collection with unique immune escape mechanism, so the IFN- $\gamma$-mediated anti-tumor immunity ${ }^{87}$ by MVs itself may be limited by the form of IFN- $\gamma$ responsive programmed cell death-Ligand 1 (PD-L1) expression on tumor cells to suppress the function of $\mathrm{T}$ cells, ${ }^{106}$ which eventually leads to immune tolerance. The strategy of MVs combined with other strategies to reverse the immune suppression could overcome the obstacle. For instance, the MVs expressing the ectodomain of programmed cell death-1 (PD-1) on the surface integrated both immune activation and blockage of the immunosuppression, leading to a significant reduction of tumor growth in colorectal cancer models. ${ }^{107}$ Based on the flexible manipulation, reversing other immunosuppressive factors such as CD47, OX40, TIM-3 and LAG-3 can also use the MVs platform to explore more combination in antitumor immunotherapy.

\section{Modification of MVs by Non-Genetic Engineering Strategy}

Considering the specific characteristics of the surface of MVs, many other non-genetic engineering modification methods can be introduced to optimize MVs for safer therapy and better antitumor effect. To selectively expose the MVs in TME, the "shielding" strategy by wrapping MVs inside highly biocompatible materials was explored, by biomineralization, chemical bonding, or membrane fusion. Considering the similar structure of outer membrane vesicle as liposomes, chemical modifications can also be introduced to modify them. However, this related work is rarely reported. Other modification strategies are summarized. For instance, Qing et al. biosynthesized a highly biocompatible calcium phosphate on the surface to encapsulate OMVs. Upon their arrival at tumor sites, the slightly acidic $\mathrm{pH}$ of TME triggered the dissolution of $\mathrm{CaP}$ shells and release MVs to activate immune response. Moreover, they further doped folic acid into $\mathrm{CaP}$ shells to enhanced the tumor targeting. ${ }^{85}$ Referred to the similar structure of various cell membranes, membrane fusion technology was used to achieve the fusion of two natural biomembranes, even the fusion of MVs with liposomes. Wang et al. ${ }^{108}$ fused MVs with the cancer cell membrane to generate a hybrid membrane, which simultaneously harnessed the homing ability of cancer cell membrane and immune response activation ability of bacterial MVs to synergistically eradicate melanoma. The strategy could be adapted to combine immunotherapy with various therapies toward different cancers by fusing the bacterial MVs with various cancer cell membranes and incorporating different therapeutic agents inside MVs (Figure 7). In addition, due to the similar lipid nature, DSPE-PEGRGD was reported to have been fused into the lipid 
A

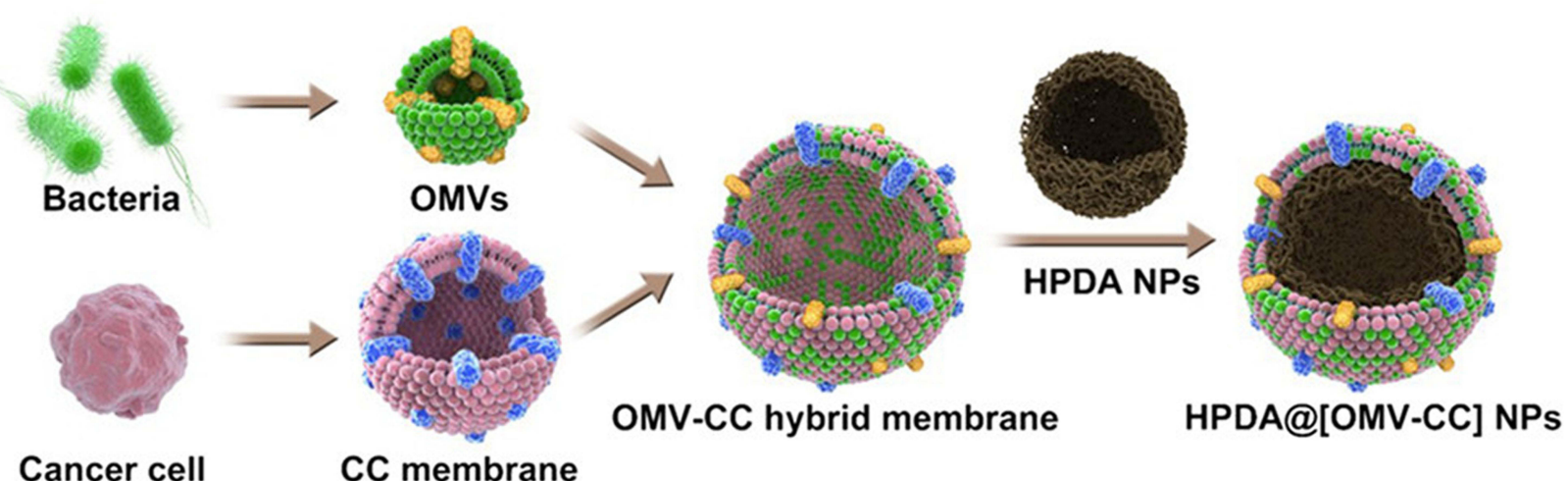

Cancer cell

B

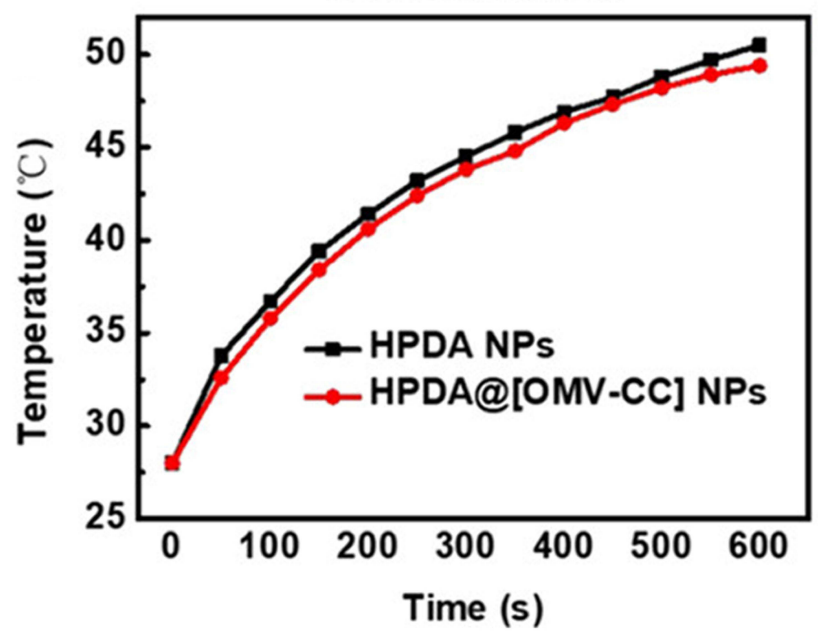

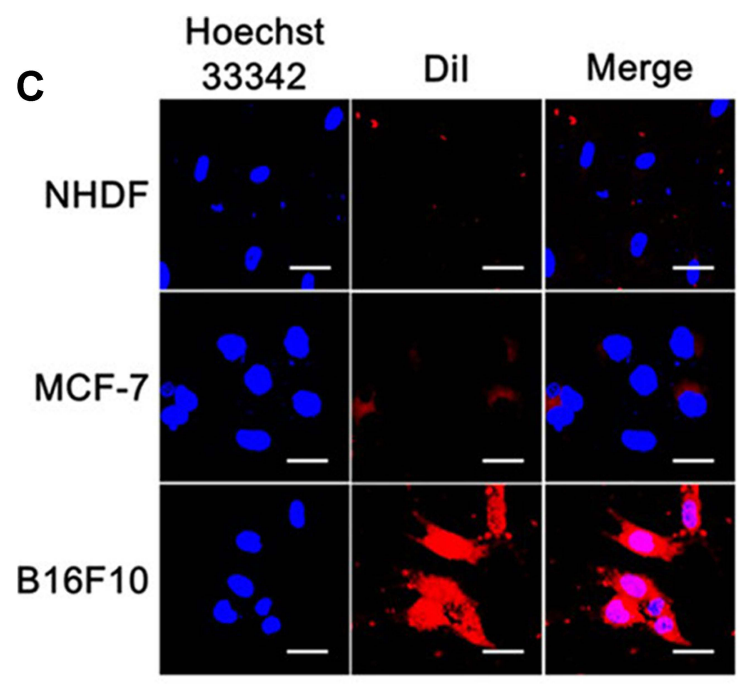

Figure 7 (A) Schematic of the membrane derived from OMV and cancer cell (CC) fusion and the resulting fused membrane camouflaged HPDA NPs to produce HPDA@ [OMV-CC] NPs. (B) Temperature elevation of HPDA NPs and HPDA@[OMV-CC] NPs (100 $\mu \mathrm{g} / \mathrm{mL})$. (C) CLSM image of NHDF cells, MCF-7 cells, and BI6-FI0 cells stained with Hoechst 33342 and cultured with Dil-dyed HPDA@[OMV-CC] NPs. Adapted with permission from Wang D, Liu C, You S, et al. Bacterial vesicle-cancer cell hybridmembrane-coated nanoparticles for tumor specific immune activationand photothermal therapy. ACS Appl Mater Interfaces. 2020;12(37):4II38-4II47. Copyright 2020 American Chemical Society. ${ }^{108}$

membrane of MVs with lipid head of DSPE by extrusion technology, ${ }^{109}$ endowing MVs with better targeting ability. The same method was also applied to coating MVs on Tegafur-loaded nanomicelles, to exert both chemotherapeutic and immunomodulatory roles for better cancer immunotherapy. ${ }^{110}$

\section{Future and Prospects}

Bacteria-related drug delivery systems (DDS) in anticancer therapy have been researched for over a century and this field has developed rapidly in recent years with advances in synthetic biology and chemistry. Different from the EPR effect of other nanoparticles, its homing ability is more conducive to penetrating physiological barriers and tumor tissues. Moreover, based on various modification strategies, the safety issues and low therapeutic efficiency have also been greatly improved, holding larger potential as auspicious candidates for therapeutic and drug-delivery applications in tumor field. ${ }^{32,54}$ From this systematic review we conclude that there will be an increasing use of bacteria-related drug delivery systems (DDS) for drug delivery in cancer treatment, along with an extensive application into various antitumor therapies. New strategies would be designed based on the previous studies, for example, we could manipulate bacteria that express specific molecules at the hypoxia tumor microenvironment via genetic manipulation, which could synergize with chemotherapeutic drug loaded in the nanomaterials for high anti-tumor efficacy.

Although the bacteria-related DDS has been widely investigated in preclinical studies, many challenges still remain in the way to further clinical application. First, the risk of the bacteria-related drug delivery system application should be 
further considered. And safer bacteria, stable conjugation between bacteria and nanoparticles, and comprehensive evaluation of their safety are needed. Next, more investigations on the controllability, stability and reproducibility of the preparation techniques should be conducted, e.g., the binding site, number of conjugated bacteria and nanoparticles, and the strength of conjugation.

Furthermore, the reports on the stability, metabolism and clearance, drug loading, retention in vivo, pharmaceutical stability during storage, pharmacokinetics (PK), biodistribution and Current Good Manufacturing Practices (cGMP) of clinical batches for this DDS were scarce, which still have a long way to go for accumulation of more data. Additionally, the standardization and regulations of the manufacturing for this DDS are less well defined due to the risk and diversity, which involves a complex set of programs and different fields, such as bioengineering and multifactorial biological processes. Deeper investigation on the intracellular mechanisms and distribution inside cells will also provide reference data for regulatory guidelines. What is more, efforts should be taken to improve the methods of in vitro characterization, which will favor the investigation on the in vitro and in vivo pharmacokinetics correlation of this DDS and promote the process of their clinical applications.

\section{Acknowledgments}

The authors would like to thank Nanjing Normal University and China Pharmaceutical University for providing supporting and facilities. The work was supported by grants from the National Natural Science Foundation of China (NO. 81972892).

\section{Disclosure}

The authors report no conflicts of interest in this work.

\section{References}

1. Couvreur P. Nanoparticles in drug delivery: past, present and future. Adv Drug Deliv Rev. 2013;65(1):21-23. doi:10.1016/j. addr.2012.04.010

2. Mohammadinejad R, Dehshahri A, Sagar Madamsetty V, et al. In vivo gene delivery mediated by non-viral vectors for cancer therapy. $J$ Control Release. 2020;325:249-275.

3. Delfi M, Sartorius R, Ashrafizadeh M, et al. Self-assembled peptide and protein nanostructures for anti-cancer therapy: targeted delivery, stimuli-responsive devices and immunotherapy. Nano Today. 2021;38.

4. Caffery B, Lee JS, Alexander-Bryant AA. Vectors for glioblastoma gene therapy: viral \& non-viral delivery strategies. Nanomaterials. 2019;9(1):105. doi:10.3390/nano9010105

5. Chang WW, Lee CH. Salmonella as an innovative therapeutic antitumor agent. Int J Mol Sci. 2014;15(8):14546-14554. doi:10.3390/ ijms 150814546
6. Panteli JT, Forbes NS. Engineered bacteria detect spatial profiles in glucose concentration within solid tumor cell masses. Biotechnol Bioeng. 2016;113(11):2474-2484. doi:10.1002/bit.26006

7. Pawelek JM, Low KB, Bermudes D. Bacteria as tumour-targeting vectors. Lancet Oncol. 2003;4(9):548-556. doi:10.1016/S14702045(03)01194-X

8. Bone RC. Toward an epidemiology and natural history of SIRS (systemic inflammatory response syndrome). JAMA. 1992;268 (24):3452-3455. doi:10.1001/jama.1992.03490240060037

9. Dinarello CA, Gelfand JA, Wolff SM. Anticytokine strategies in the treatment of the systemic inflammatory response syndrome. JAMA. 1993;269(14):1829-1835. doi:10.1001/jama.1993.03500140081040

10. Gericke D, Engelbart K. Oncolysis by Clostridia. ii. experiments on a tumor spectrum with a variety of clostridia in combination with heavy metal. Cancer Res. 1964;24:217-221.

11. Singh AV, Sitti M. Targeted drug delivery and imaging using mobile milli/microrobots: a promising future towards theranostic pharmaceutical design. Curr Pharm Des. 2016;22 (11):1418-1428. doi:10.2174/1381612822666151210124326

12. Cao ZP, Liu JY. Bacteria and bacterial derivatives as drug carriers for cancer therapy. $J$ Control Release. 2020;326:396-407. doi:10.1016/j.jconrel.2020.07.009

13. DeWeerdt S. BACTERIOLOGY A caring culture. Nature. 2013;504(7480):S4-S5. doi:10.1038/504S4a

14. Prout GR. Intracavitary bacillus Calmette-Guerin in the treatment of superficial bladder tumors - comment. J Urol. 2002;167 (2):893-894.

15. Prout GR. Intracavitary bacillus Calmette-Guerin in the treatment of superficial bladder tumors comment. J Urol. 2017;197(2): S144-S145.

16. Biot C, Rentsch CA, Gsponer JR, et al. Preexisting BCG-specific $\mathrm{T}$ cells improve intravesical immunotherapy for bladder cancer. $S c i$ Transl Med. 2012;4(137):137ra172. doi:10.1126/scitranslmed.3 003586

17. Clairmont $\mathrm{C}$, Lee KC, Pike J, et al. Biodistribution and genetic stability of the novel antitumor agent VNP20009, a genetically modified strain of Salmonella typhimurium. $J$ Infect Dis. 2000;181(6):1996-2002. doi:10.1086/315497

18. Toso JF, Gill VJ, Hwu P, et al. Phase I study of the intravenous administration of attenuated Salmonella typhimurium to patients with metastatic melanoma. J Clin Oncol. 2002;20(1):142-152. doi:10.1200/JCO.2002.20.1.142

19. Staedtke V, Roberts NJ, Bai RY, Zhou S. Clostridium novyi-NT in cancer therapy. Genes Dis. 2016;3(2):144-152. doi:10.1016/j. gendis.2016.01.003

20. Agrawal N, Bettegowda C, Cheong I, et al. Bacteriolytic therapy can generate a potent immune response against experimental tumors. Proc Natl Acad Sci U S A. 2004;101(42):15172. doi:10.1073/pnas.0406242101

21. Staedtke V, Bai R-Y, Sun W, et al. Clostridium novyi-NT can cause regression of orthotopically implanted glioblastomas in rats. Oncotarget. 2015;6(8):5536-5546.

22. Gniadek TJ, Augustin L, Schottel J, Leonard A, Batist G. A phase I, dose escalation, single dose trial of oral attenuated Salmonella typhimurium containing human IL-2 in patients with metastatic gastrointestinal cancers. J Immunother. 2020;43(7):217-221. doi:10.1097/CJI.0000000000000325

23. Flickinger JC Jr, Rodeck U, Snook AE. Listeria monocytogenes as a vector for cancer immunotherapy: current understanding and progress. Vaccines. 2018;6(3):48. doi:10.3390/vaccines6030048

24. Gunn GR, Zubair A, Peters C, Pan ZK, Wu TC, Paterson Y. Two Listeria monocytogenes vaccine vectors that express different molecular forms of human papilloma virus-16 (HPV-16) E7 induce qualitatively different $\mathrm{T}$ cell immunity that correlates with their ability to induce regression of established tumors immortalized by HPV-16. J Immunol. 2001;167(11):6471-6479. 
25. Shahabi V, Reyes-Reyes M, Wallecha A, Rivera S, Paterson Y, Maciag P. Development of a Listeria monocytogenes based vaccine against prostate cancer. Cancer Immunol Immunother. 2008;57(9):1301-1313. doi:10.1007/s00262-008-0463-z

26. Singh R, Dominiecki ME, Jaffee EM, Paterson Y. Fusion to Listeriolysin $\mathrm{O}$ and delivery by Listeria monocytogenes enhances the immunogenicity of HER-2/neu and reveals subdominant epitopes in the FVB/N mouse. J Immunol. 2005;175(6):3663-3673. doi:10.4049/jimmunol.175.6.3663

27. Brahmer JR, Johnson ML, Cobo M, et al. JNJ-64041757 (JNJ757), a live, attenuated, double-deleted Listeria monocytogenesbased immunotherapy in patients with NSCLC: results from two phase 1 studies. JTO Clin Res Rep. 2021;2(2):100103. doi:10.1016/j.jtocrr.2020.100103

28. Leventhal DS, Sokolovska A, Li N, et al. Immunotherapy with engineered bacteria by targeting the STING pathway for anti-tumor immunity. Nat Commun. 2020;11(1):2739. doi:10.1038/s41467-020-16602-0

29. Lee BK, Yun YH, Park K. Smart nanoparticles for drug delivery: boundaries and opportunities. Chem Eng Sci. 2015;125:158-164. doi:10.1016/j.ces.2014.06.042

30. Yan X, Zhou Q, Vincent M, et al. Multifunctional biohybrid magnetite microrobots for imaging-guided therapy. Sci Robot. 2017;2(12). doi:10.1126/scirobotics.aaq1155

31. Chen F, Zang Z, Chen Z, et al. Nanophotosensitizer-engineered Salmonella bacteria with hypoxia targeting and photothermal-assisted mutual bioaccumulation for solid tumor therapy. Biomaterials. 2019;214:119226. doi:10.1016/j. biomaterials.2019.119226

32. Luo $\mathrm{Y}, \mathrm{Xu} \mathrm{D}$, Gao $\mathrm{X}$, et al. Nanoparticles conjugated with bacteria targeting tumors for precision imaging and therapy. Biochem Biophys Res Commun. 2019;514(4):1147-1153. doi:10.1016/j.bbrc.2019.05.074

33. Chen QW, Liu XH, Fan JX, et al. Self-mineralized photothermal bacteria hybridizing with mitochondria-targeted metal-organic frameworks for augmenting photothermal tumor therapy. $A d v$ Funct Mater. 2020;30(14):1909806.

34. Felfoul O, Mohammadi M, Taherkhani S, et al. Magnetoaerotactic bacteria deliver drug-containing nanoliposomes to tumour hypoxic regions. Nat Nanotechnol. 2016;11 (11):941-947. doi:10.1038/nnano.2016.137

35. Taherkhani S, Mohammadi M, Daoud J, Martel S, Tabrizian M. Covalent binding of nanoliposomes to the surface of magnetotactic bacteria for the synthesis of self-propelled therapeutic agents. ACS Nano. 2014;8(5):5049-5060. doi:10.1021/ nn5011304

36. Kuru E, Hughes HV, Brown PJ, et al. In situ probing of newly synthesized peptidoglycan in live bacteria with FluorescentDamino acids. Angew Chem Int Ed. 2012;51(50):12519-12523. doi:10.1002/anie.201206749

37. Moreno VM, Alvarez E, Izquierdo-Barba I, Baeza A, SerranoLopez J, Vallet-Regi M. Bacteria as nanoparticles carrier for enhancing penetration in a tumoral matrix model. Adv Mater Interfaces. 2020;7(11):1901942.

38. Wu M, Wu W, Duan Y, Li X, Qi G, Liu B. Photosensitizerbacteria biohybrids promote photodynamic cancer cell ablation and intracellular protein delivery. Chem Mater. 2019;31 (18):7212-7220. doi:10.1021/acs.chemmater.9b01518

39. Hu Q, Wu M, Fang $\mathrm{C}$, et al. Engineering nanoparticle-coated bacteria as oral DNA vaccines for cancer immunotherapy. Nano Lett. 2015;15(4):2732-2739. doi:10.1021/acs. nanolett. $5 \mathrm{~b} 00570$

40. Chen J, Shen C, Zheng C, et al. Study of properties of VEGFR2 active site and binding mode of VEGFR2 and its inhibitors. Acta Chim Sin. 2007;65(6):547-552.
41. Luo $\mathrm{CH}$, Huang $\mathrm{CT}$, Su $\mathrm{CH}$, Yeh $\mathrm{CS}$. Bacteria-mediated hypoxia-specific delivery of nanoparticles for tumors imaging and therapy. Nano Lett. 2016;16(6):3493-3499. doi:10.1021/acs. nanolett.6b00262

42. Wang XY, Cao ZP, Zhang MM, Meng L, Ming ZZ, Liu JY. Bioinspired oral delivery of gut microbiota by self-coating with biofilms. Sci Adv. 2020;6(26):eabb1952.

43. Imberti C, Zhang P, Huang H, Sadler PJ. New designs for phototherapeutic transition metal complexes. Angew Chem Int Ed Engl. 2020;59(1):61-73. doi:10.1002/anie.201905171

44. De Windt W, Aelterman P, Verstraete W. Bioreductive deposition of palladium (0) nanoparticles on Shewanella oneidensis with catalytic activity towards reductive dechlorination of polyschlorinated biphenyls. Environ Microbiol. 2005;7(3):314-325. doi:10.1111/j.1462-2920.2005.00696.x

45. Chen X, Shi S, Wei J, Chen M, Zheng N. Two-dimensional Pd-based nanomaterials for bioapplications. Sci Bull. 2017;62 (8):579-588. doi:10.1016/j.scib.2017.02.012

46. Wang X-N, Niu M-T, Fan J-X, Chen Q-W, Zhang X-Z. Photoelectric bacteria enhance the in situ production of tetrodotoxin for antitumor therapy. Nano Lett. 2021;21(10):4270-4279. doi:10.1021/acs.nanolett.1c00408

47. Yan S, Zeng X, Wang Y, Liu BF. Biomineralization of bacteria by a metal-organic framework for therapeutic delivery. $A d v$ Healthcare Mater. 2020;9(12):e2000046. doi:10.1002/ adhm.202000046

48. Chu C, Su M, Zhu J, et al. Metal-organic framework nanoparticle-based biomineralization: a new strategy toward cancer treatment. Theranostics. 2019;9(11):3134-3149. doi:10.7150/ thno.33539

49. Zheng S, Han J-W, Seong Y, Choi Y, Jin P. Active tumor-therapeutic liposomal bacteriobot combining a drug (paclitaxel)-encapsulated liposome with targeting bacteria (Salmonella Typhimurium). Sens Actuators B Chem. 2016;224:217-224. doi:10.1016/j.snb.2015.09.034

50. Traore MA, Damico CM, Behkam B. Biomanufacturing and self-propulsion dynamics of nanoscale bacteria-enabled autonomous delivery systems. Appl Phys Lett. 2014;105(17):121. doi:10.1063/1.4900641

51. Goldenberg DM, Sharkey RM, Paganelli G, Barbet J, Chatal JF. Antibody pretargeting advances cancer radioimmunodetection and radioimmunotherapy. J Clin Oncol. 2006;24(5):823-834. doi:10.1200/JCO.2005.03.8471

52. Park SJ, Park SH, Cho S, et al. New paradigm for tumor theranostic methodology using bacteria-based microrobot. Sci Rep. 2013;3:3394. doi:10.1038/srep03394

53. Sahari A, Traore MA, Scharf BE, Behkam B. Directed transport of bacteria-based drug delivery vehicles: bacterial chemotaxis dominates particle shape. Biomed Microdevices. 2014;16 (5):717-725. doi:10.1007/s10544-014-9876-y

54. Suh S, Jo A, Traore MA, et al. Nanoscale Bacteria-Enabled Autonomous Drug Delivery System (NanoBEADS) enhances intratumoral transport of nanomedicine. Adv Sci. 2019;6 (3): 1801309 .

55. Uthaman S, Zheng S, Han J, et al. Preparation of engineered Salmonella Typhimurium-driven hyaluronic-acid-based microbeads with both chemotactic and biological targeting towards breast cancer cells for enhanced anticancer therapy. Adv Healthcare Mater. 2016;5(2):288-295. doi:10.1002/adhm.201500556

56. Kojima M, Zhang Z, Nakajima M, Ooe K, Fukuda T. Construction and evaluation of bacteria-driven liposome. Sens Actuators B Chem. 2013;183:395-400. doi:10.1016/j. snb.2013.03.127

57. Zhang Z, Li Z, Yu W, Li K, Xie Z, Shi Z. Propulsion of liposomes using bacterial motors. Nanotechnology. 2013;24(18):185103. doi:10.1088/0957-4484/24/18/185103 
58. Cho S, Park SJ, Ko SY, Park JO, Park S. Development of bacteria-based microrobot using biocompatible poly(ethylene glycol). Biomed Microdevices. 2012;14(6):1019-1025. doi:10.1007/s10544-012-9704-1

59. Rabanel JM, Hildgen P, Banquy X. Assessment of PEG on polymeric particles surface, a key step in drug carrier translation. J Control Release. 2014;185:71-87. doi:10.1016/j. jconrel.2014.04.017

60. Kolate A, Baradia D, Patil S, Vhora I, Kore G, Misra A. PEG a versatile conjugating ligand for drugs and drug delivery systems. J Control Release. 2014;192:67-81. doi:10.1016/j. jconrel.2014.06.046

61. Hosseinidoust Z, Mostaghaci B, Yasa O, Park BW, Singh AV, Sitti M. Bioengineered and biohybrid bacteria-based systems for drug delivery. Adv Drug Deliv Rev. 2016;106(Pt A):27-44.

62. Fox ME, Lemmon MJ, Mauchline ML, et al. Anaerobic bacteria as a delivery system for cancer gene therapy: in vitro activation of 5-fluorocytosine by genetically engineered clostridia. Gene Ther. 1996;3(2):173-178.

63. Moese JR, Moese G. Oncolysis by clostridia. i. activity of clostridium butyricum (M-55) and other nonpathogenic clostridia against the Ehrlich carcinoma. Cancer Res. 1964;24:212-216.

64. Gurbatri CR, Lia I, Vincent R, et al. Engineered probiotics for local tumor delivery of checkpoint blockade nanobodies. Sci Transl Med. 2020;12(530). doi:10.1126/scitranslmed.aax0876

65. Din MO, Danino T, Prindle A, et al. Synchronized cycles of bacterial lysis for in vivo delivery. Nature. 2016;536 (7614):81-85. doi:10.1038/nature18930

66. Fan C, Davison PA, Habgood R, et al. Chromosome-free bacterial cells are safe and programmable platforms for synthetic biology. Proc Natl Acad Sci. 2020;117(12):6752. doi:10.1073/ pnas. 1918859117

67. Schwechheimer C, Kuehn MJ. Outer-membrane vesicles from gram-negative bacteria: biogenesis and functions. Nat Rev Microbiol. 2015;13(10):605-619. doi:10.1038/nrmicro3525

68. Brown L, Wolf JM, Prados-Rosales R, Casadevall A. Through the wall: extracellular vesicles in gram-positive bacteria, mycobacteria and fungi. Nat Rev Microbiol. 2015;13(10):620-630. doi:10.1038/nrmicro3480

69. Orench-Rivera N, Kuehn MJ. Environmentally controlled bacterial vesicle-mediated export. Cell Microbiol. 2016;18 (11):1525-1536. doi:10.1111/cmi.12676

70. Clayton A, Harris CL, Court J, Mason MD, Morgan BP. Antigenpresenting cell exosomes are protected from complement-mediated lysis by expression of CD55 and CD59. Eur J Immunol. 2003;33 (2):522-531. doi:10.1002/immu.200310028

71. Peng LH, Wang MZ, Chu Y, et al. Engineering bacterial outer membrane vesicles as transdermal nanoplatforms for photo-TRAIL-programmed therapy against melanoma. Sci Adv. 2020;6(27):eaba2735. doi:10.1126/sciadv.aba2735

72. Torchilin VP. Passive and active drug targeting: drug delivery to tumors as an example. Handb Exp Pharmacol. 2010;197:3-53.

73. MacDiarmid JA, Langova V, Bailey D, et al. Targeted doxorubicin delivery to brain tumors via minicells: proof of principle using dogs with spontaneously occurring tumors as a model. PLoS One. 2016;11(4):e0151832. doi:10.1371/journal. pone. 0151832

74. Solomon BJ, Desai J, Rosenthal M, et al. A first-time-in-human Phase I clinical trial of bispecific antibody-targeted, paclitaxel-packaged bacterial minicells. PLoS One. 2015;10(12): e0144559. doi:10.1371/journal.pone.0144559

75. Yu B, Tai HC, Xue W, Lee LJ, Lee RJ. Receptor-targeted nanocarriers for therapeutic delivery to cancer. Mol Membr Biol. 2010;27(7):286-298. doi:10.3109/09687688.2010.521200
76. Sagnella SM, Yang L, Stubbs GE, et al. Cyto-immuno-therapy for cancer: a pathway elicited by tumor-targeted, cytotoxic drug-packaged bacterially derived nanocells. Cancer Cell. 2020;37(3):354-370.e357. doi:10.1016/j.ccell.2020.02.001

77. Sagnella SM, Trieu J, Brahmbhatt $H$, et al. Targeted doxorubicin-loaded bacterially derived nano-cells for the treatment of neuroblastoma. Mol Cancer Ther. 2018;17 (5):1012-1023. doi:10.1158/1535-7163.MCT-17-0738

78. Alfaleh MA, Howard CB, Sedliarou I, et al. Targeting mesothelin receptors with drug-loaded bacterial nanocells suppresses human mesothelioma tumour growth in mouse xenograft models. PLoS One. 2017;12(10):e0186137. doi:10.1371/journal. pone. 0186137

79. Whittle JR, Lickliter JD, Gan HK, et al. First in human nanotechnology doxorubicin delivery system to target epidermal growth factor receptors in recurrent glioblastoma. $J$ Clin Neurosci. 2015;22(12):1889-1894. doi:10.1016/j.jocn.2015.06.005

80. MacDiarmid JA, Mugridge NB, Weiss JC, et al. Bacterially derived $400 \mathrm{~nm}$ particles for encapsulation and cancer cell targeting of chemotherapeutics. Cancer Cell. 2007;11(5):431-445. doi:10.1016/j.ccr.2007.03.012

81. Gujrati V, Prakash J, Malekzadeh-Najafabadi J, et al. Bioengineered bacterial vesicles as biological nano-heaters for optoacoustic imaging. Nat Commun. 2019;10(1):1114. doi:10.1038/s41467-019-09034-y

82. Riley PA. Melanin. Int $J$ Biochem Cell Biol. 1997;29 (11):1235-1239. doi:10.1016/S1357-2725(97)00013-7

83. Liu Y, Ai K, Liu J, Deng M, He Y, Lu L. Dopamine-melanin colloidal nanospheres: an efficient near-infrared photothermal therapeutic agent for in vivo cancer therapy. Adv Mater. 2013;25(9):1353-1359. doi:10.1002/adma.201204683

84. Kaparakis-Liaskos M, Ferrero RL. Immune modulation by bacterial outer membrane vesicles. Nat Rev Immunol. 2015;15 (6):375-387. doi:10.1038/nri3837

85. Qing S, Lyu C, Zhu L, et al. Biomineralized bacterial outer membrane vesicles potentiate safe and efficient tumor microenvironment reprogramming for anticancer therapy. Adv Mater. 2020;32(47):e2002085. doi:10.1002/adma.202002085

86. Gujrati V, Kim S, Kim SH, et al. Bioengineered bacterial outer membrane vesicles as cell-specific drug-delivery vehicles for cancer therapy. ACS Nano. 2014;8(2):1525-1537. doi:10.1021/nn405724x

87. Kim OY, Park HT, Dinh NTH, et al. Bacterial outer membrane vesicles suppress tumor by interferon- $\gamma$-mediated antitumor response. Nat Commun. 2017;8(1):626. doi:10.1038/s41467-017-00729-8

88. Kato K, Omura H, Ishitani R, Nureki O. Cyclic GMP-AMP as an endogenous second messenger in innate immune signaling by cytosolic DNA. Annu Rev Biochem. 2017;86:541-566. doi:10.1146/annurev-biochem-061516-044813

89. Li RZ, Liu Q. Engineered bacterial outer membrane vesicles as multifunctional delivery platforms. Front Mater. 2020;7. doi:10.3389/fmats.2020.00202

90. Lee SY, Choi JH, Xu Z. Microbial cell-surface display. Trends Biotechnol. 2003;21(1):45-52. doi:10.1016/S0167-7799(02)00006-9

91. Gerritzen MJH, Martens DE, Wijffels RH, van der Pol L, Stork M. Bioengineering bacterial outer membrane vesicles as vaccine platform. Biotechnol Adv. 2017;35(5):565-574. doi:10.1016/j.biotechadv.2017.05.003

92. Baslé A, Rummel G, Storici P, Rosenbusch JP, Schirmer T. Crystal structure of osmoporin OmpC from E. coli at 2.0 A. J Mol Biol. 2006;362(5):933-942. doi:10.1016/j.jmb.2006.08.002

93. Pautsch A, Schulz GE. Structure of the outer membrane protein A transmembrane domain. Nat Struct Biol. 1998;5 (11):1013-1017. doi:10.1038/2983

94. Kim JY, Doody AM, Chen DJ, et al. Engineered bacterial outer membrane vesicles with enhanced functionality. $J \mathrm{Mol}$ Biol. 2008;380(1):51-66. doi:10.1016/j.jmb.2008.03.076 
95. Gao J, Wang S, Dong X, Wang Z. RGD-expressed bacterial membrane-derived nanovesicles enhance cancer therapy via multiple tumorous targeting. Theranostics. 2021;11(7):3301-3316. doi: $10.7150 /$ thno. 51988

96. Russo AJ, Behl B, Banerjee I, Rathinam VAK. Emerging insights into noncanonical inflammasome recognition of microbes. $J \mathrm{Mol}$ Biol. 2018;430(2):207-216. doi:10.1016/j.jmb.2017.10.003

97. Hayashi F, Smith KD, Ozinsky A, et al. The innate immune response to bacterial flagellin is mediated by toll-like receptor 5 . Nature. 2001;410(6832):1099-1103. doi:10.1038/35074106

98. Oliveira-Nascimento L, Massari P, Wetzler LM. The role of TLR2 in infection and immunity. Front Immunol. 2012;3:79. doi:10.3389/fimmu.2012.00079

99. Kuzmich NN, Sivak KV, Chubarev VN, Porozov YB, SavateevaLyubimova TN, Peri F. TLR4 signaling pathway modulators as potential therapeutics in inflammation and sepsis. Vaccines. 2017;5(4):34.

100. Carleton HA, Lara-Tejero M, Liu X, Galán JE. Engineering the type III secretion system in non-replicating bacterial minicells for antigen delivery. Nat Commun. 2013;4(1):1590. doi:10.1038/ ncomms 2594

101. Huang W, Shu C, Hua L, et al. Modified bacterial outer membrane vesicles induce autoantibodies for tumor therapy. Acta Biomater. 2020;108:300-312. doi:10.1016/j.actbio.2020.03.030

102. Wang S, Huang W, Li K, et al. Engineered outer membrane vesicle is potent to elicit HPV16E7-specific cellular immunity in a mouse model of TC-1 graft tumor. Int $J$ Nanomedicine. 2017;12:6813-6825. doi:10.2147/IJN.S143264

103. Zakeri B, Fierer JO, Celik E, et al. Peptide tag forming a rapid covalent bond to a protein, through engineering a bacterial adhesin. Proc Natl Acad Sci U S A. 2012;109(12):E690-E697. doi:10.1073/pnas.1115485109
104. Veggiani G, Nakamura T, Brenner MD, et al. Programmable polyproteams built using twin peptide superglues. Proc Natl Acad Sci U S A. 2016;113(5):1202-1207. doi:10.1073/pnas.1519214113

105. Cheng K, Zhao R, Li Y, et al. Bioengineered bacteria-derived outer membrane vesicles as a versatile antigen display platform for tumor vaccination via plug-and-display technology. Nat Commun. 2021;12(1):2041. doi:10.1038/s41467-021-22308-8

106. Garcia-Diaz A, Shin DS, Moreno BH, et al. Interferon receptor signaling pathways regulating PD-L1 and PD-L2 expression. Cell Rep. 2019;29(11):3766. doi:10.1016/j.celrep.2019.11.113

107. Li Y, Zhao R, Cheng K, et al. Bacterial outer membrane vesicles presenting programmed death 1 for improved cancer immunotherapy via immune activation and checkpoint Inhibition. ACS Nano. 2020;14(12):16698-16711. doi:10.1021/acsnano.0c03776

108. Wang D, Liu C, You S, et al. Bacterial vesicle-cancer cell hybrid membrane-coated nanoparticles for tumor specific immune activation and photothermal therapy. ACS Appl Mater Interfaces. 2020;12 (37):41138-41147. doi:10.1021/acsami.0c13169

109. Kulp A, Kuehn MJ. Biological functions and biogenesis of secreted bacterial outer membrane vesicles. Annu Rev Microbiol. 2010;64 (1):163-184. doi:10.1146/annurev.micro.091208.073413

110. Chen Q, Bai $\mathrm{H}, \mathrm{Wu} \mathrm{W}$, et al. Bioengineering bacterial vesicle-coated polymeric nanomedicine for enhanced cancer immunotherapy and metastasis prevention. Nano Lett. 2020;20 (1):11-21. doi:10.1021/acs.nanolett.9b02182
International Journal of Nanomedicine

\section{Publish your work in this journal}

The International Journal of Nanomedicine is an international, peerreviewed journal focusing on the application of nanotechnology in diagnostics, therapeutics, and drug delivery systems throughout the biomedical field. This journal is indexed on PubMed Central, MedLine, CAS, SciSearch ${ }^{\mathbb{}}$, Current Contents ${ }^{\mathbb{R}} /$ Clinical Medicine, $^{2}$

\section{Dovepress}

Journal Citation Reports/Science Edition, EMBase, Scopus and the Elsevier Bibliographic databases. The manuscript management system is completely online and includes a very quick and fair peer-review system, which is all easy to use. Visit http://www.dovepress.com/ testimonials.php to read real quotes from published authors. 